\title{
Multivalent Nanoparticle-Based Vaccines Protect Hamsters Against SARS-CoV-2 After a Single Immunization
}

\section{Shiho Chiba}

University of Wisconsin-Madison

\section{Steven J. Frey}

Georgia Institute of Technology

Peter J. Halfmann

University of Wisconsin-Madison

\section{Makoto Kuroda}

University of Wisconsin-Madison

\section{Tadashi Maemura}

University of Wisconsin-Madison

Yoshihiro Kawaoka ( $\nabla$ yoshihiro.kawaoka@wisc.edu )

University of Wisconsin-Madison

Ravi S. Kane ( $\nabla$ ravi.kane@chbe.gatech.edu )

Georgia Institute of Technology

\section{Research Article}

Keywords: vaccine, SARS-CoV-2, nanoparticle, virus-like particle, Spike

Posted Date: December 10th, 2020

DOl: https://doi.org/10.21203/rs.3.rs-121208/v1

License: (9) This work is licensed under a Creative Commons Attribution 4.0 International License. Read Full License

Version of Record: A version of this preprint was published at Communications Biology on December 10th, 2020. See the published version at https://doi.org/10.1038/s42003-021-02128-8. 
1 Multivalent Nanoparticle-Based Vaccines Protect Hamsters Against SARS-CoV-2 After a Single 2 Immunization

3

4 Shiho Chiba ${ }^{1, \dagger}$, Steven J. Frey ${ }^{2, \dagger}$, Peter J. Halfmann ${ }^{1}$, Makoto Kuroda' ${ }^{1}$, Tadashi Maemura $^{1}$, Yoshihiro Kawaoka ${ }^{1 *}$, 5 Ravi S. Kane $2, *$

6

$7 \quad{ }^{1}$ Influenza Research Institute, Department of Pathobiological Sciences, School of Veterinary Medicine, University 8 of Wisconsin, Madison, WI, 53711, USA

9

$10{ }^{2}$ School of Chemical \& Biomolecular Engineering, Georgia Institute of Technology, Atlanta, Georgia, 30332, USA 11

$12+$ Equal contribution

13

14 * To whom correspondence should be addressed:

15 yoshihiro.kawaoka@wisc.edu, ravi.kane@chbe.gatech.edu 
The COVID-19 pandemic continues to wreak havoc as worldwide SARS-CoV-2 infection, hospitalization, and death rates climb unabated. Effective vaccines remain the most promising approach to counter SARS-CoV-2. Yet, while promising results are emerging from COVID-19 vaccine trials, the need for multiple doses and the challenges associated with the widespread distribution and administration of vaccines remain concerns. Here, we engineered the coat protein of the MS2 bacteriophage ${ }^{1,2}$ and generated nanoparticles displaying multiple copies of the SARS-CoV-2 spike (S) protein. The use of these nanoparticles as vaccines generated high neutralizing antibody titers and protected Syrian hamsters $^{3}$ from a challenge with SARS-CoV-2 after a single immunization with no infectious virus detected in the lungs. This nanoparticle-based vaccine platform thus provides protection after a single immunization and may be broadly applicable for protecting against SARS-CoV-2 and future pathogens with pandemic potential.

The COVID-19 pandemic continues to rage worldwide with more than a million estimated fatalities already and global economic costs in the hundreds of billions of dollars. Without an effective vaccine, SARSCoV-2 will continue to strain the world's economies and devastate many facets of our society. Various vaccines such as nucleic acid-based vaccines, viral vector-based vaccines, subunit vaccines, and inactivated vaccines are in different stages of clinical trials ${ }^{4}$. The contemporary vaccine candidates focus on stimulating protective immune responses to the spike (S) protein of SARS-CoV-2, the protein that facilitates viral entry by binding to the angiotensin-converting enzyme 2 (ACE2) receptor on the surface of host cells ${ }^{5}$. Neutralizing antibodies that target the spike protein could, therefore, play a role in protecting the host from this viral infection ${ }^{6,7}$.

Although these standard vaccine platforms may provide the first generation of vaccines against SARSCoV-2, nanotechnology ${ }^{8}$ has the potential to offer new and improved vaccine platforms against diseases caused by emerging viruses including SARS-CoV-2. Nanoparticles such as virus-like particles (VLPs) are ideal scaffolds for antigen display, because they emulate many of the properties of natural viruses including their size and 
1 geometry ${ }^{1,8-10}$. Moreover, the multivalent display of antigens from nanoscale scaffolds can result in the effective

2 clustering of B cell receptors and greatly enhance their immunogenicity ${ }^{11}$. Indeed, a recent report confirmed that

3 the S protein displayed on a nanoscale scaffold was more immunogenic in mice than the S protein administered

4 alone, but the study used two sequential immunizations (prime + boost) and did not test protective efficacy in

5 mice challenged with SARS-CoV-2 ${ }^{12}$. In the present study, our goal was to create a general platform for nanoparticle-based antigen display that could provide protection against SARS-CoV-2 after a single

7 immunization.

8 Generation and in vitro Characterization of Nanoparticle-based Vaccines

9 We first sought to develop a general platform for the VLP-based multivalent display of the S protein of SARSCoV-2. VLPs comprise coat proteins that self-assemble to form repetitive, dense arrays of antigen that emulate the size and geometry of natural viruses ${ }^{9}$. We generated VLPs coated with streptavidin (SA) that display biotinylated antigens, such as biotinylated SARS-CoV-2 S protein (Figure 1a), based on the very high affinity biotin-streptavidin interaction.

Specifically, we generated VLPs based on the coat protein of the RNA bacteriophage MS2 ${ }^{1,2}$. MS2 consists of 180 monomeric coat proteins that self-assemble to form an icosahedral structure consisting of 90 homodimers that measures approximately $27 \mathrm{~nm}$ in diameter. Peabody et al. generated a variant of the MS2 coat protein in which the two subunits of the dimer were genetically fused and found that a surface loop on this single-chain dimer could tolerate the insertion of a peptide ${ }^{13}$. Accordingly, we generated a single-chain MS2 coat protein dimer wherein the second monomer had an AviTag inserted in this surface loop. The inserted AviTag allows for site-specific biotinylation by the enzyme BirA. DNA encoding this MS2-AviTag construct was coexpressed with BirA in BL21(DE3) competent Escherichia coli (E. coli) cells. Following expression, the cells were lysed and the MS2-AviTag was purified by using HiScreen Capto Core 700 columns and size exclusion chromatography (SEC). The purified MS2-AviTag was partially biotinylated due to its co-expression with BirA. A commercially available kit was then used to further biotinylate the MS2-AviTag in vitro, which resulted in near as inclusion bodies, refolded, and purified using Iminobiotin Affinity Chromatography (IBAC). The resulting MS2SA VLPs were separated from the excess SA through SEC, and the purified MS2-SA VLPs were characterized 
1 by analytical SEC (Figure 1b), and dynamic light scattering (DLS) (Figure 1c). This characterization revealed 2 that the purified MS2-SA VLPs were uniform in size and approximately $50 \mathrm{~nm}$ in diameter.

We next generated a biotinylated variant of the SARS-CoV-2 S protein that could be displayed on the MS2-SA VLPS. Wrapp et al. recently reported a prefusion-stabilized variant of the SARS-CoV-2 S protein, S$2 \mathrm{P}$, which contains 2 proline substitutions ${ }^{14}$. To make a version of this variant that was compatible with display on the MS2-SA VLPs, we created plasmids encoding the stabilized prefusion S ectodomain with a C-terminal

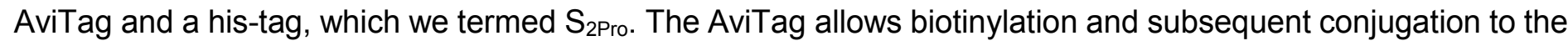
VLPs, whereas the his-tag allows purification by use of immobilized metal affinity chromatography (IMAC). We expressed the $S_{2 \text { Pro }}$ protein in Expi293F cells and purified the secreted protein from the cell culture media by using IMAC. The protein was then biotinylated enzymatically in vitro by BirA. Finally, the protein was separated from BirA and other impurities by using SEC and characterized by use of sodium dodecyl sulfate-polyacrylamide gel electrophoresis (SDS-PAGE) (Fig. 2a).

The purified, biotinylated $S_{2 P r o}$ protein was then mixed with the MS2-SA VLPs to form VLP-S 2 Pro. SDSPAGE characterization of the VLP-S 2 Pro revealed the expected three distinct bands (Figure 2a): the upper band runs alongside $S$ protein alone and appears at $\sim 140 \mathrm{kDa}$, which corresponds to the approximate molecular weight of a single monomer of the $S$ trimer; the middle band appears at the molecular weight of an MS2 coat protein dimer ( $\sim 29 \mathrm{kDa})$; and the lower band corresponds to the molecular weight of a monomer of SA $(\sim 14$ $\mathrm{kDa}$ ). This characterization indicates that the VLP-S $\mathrm{S}_{2 \mathrm{Pro}}$ is pure and consists of only $\mathrm{S}_{2 \mathrm{Pro}}$, SA, and MS2. The VLP-S ${ }_{2 P r o}$ construct was further characterized by using analytical SEC (Figure $2 b$ ). The UV trace of the VLP$\mathrm{S}_{2 \text { Pro }}$ is represented by a solid line, which appears as a single peak with no trailing shoulder. The lack of a trailing shoulder suggests that there is little to no unbound $S_{2 \text { Pro }}$ protein in the VLP-S 2 Pro solution, as the UV trace of the $\mathrm{S}_{2 \text { Pro }}$ protein alone results is a single peak that slightly trails the peak of the VLP-S and is represented by a dashed line. Furthermore, the locations of the peaks are consistent with the constructs' size relative to the size of the molecular weight standard thyroglobulin $(660 \mathrm{kDa})$. The location at which thyroglobulin elutes is represented by a vertical gray line. To more precisely quantify the size of the VLP-S ${ }_{2 \text { Pro }}$ constructs, we used DLS (Figure 2c). DLS measurements indicated that the VLP-S 2 Pro constructs had a relatively uniform size distribution (as represented by the solid line) with a diameter slightly smaller than $100 \mathrm{~nm}$. Given that the MS2-SA has a 
1 diameter of approximately $50 \mathrm{~nm}$ (Figure 1c) and the S protein (with the trimerization domain and C-terminal 2 AviTag) would theoretically be approximately $20 \mathrm{~nm}$ in length, the $\sim 100 \mathrm{~nm}$ measurement for the VLP-S is

reasonable. Finally, to ensure that the S proteins remained properly folded after conjugation to the VLPS, we assessed the binding of ACE2-Fc and the receptor-binding domain (RBD)-binding monoclonal antibody CR3022 to $\mathrm{S}_{2 \mathrm{Pro}}$ protein alone and to VLP-S 2 Pro $($ Figure $2 \mathrm{~d}$ ). ACE2 is the cellular receptor for SARS-CoV-2 and binds to the receptor binding motif of the $S$ protein ${ }^{15}$. A common mechanism of SARS-CoV-2 neutralization is the inhibition of S protein binding to ACE2, so it is important to demonstrate that the ACE2 binding site is properly folded $^{16,17}$. CR3022 is a neutralizing antibody that binds to the S protein RBD outside of the ACE2 binding site ${ }^{16,18}$. ELISA showed that both ACE2-Fc and CR3022 can bind to the $\mathrm{S}_{2 \text { Pro }}$ protein alone and to VLP-S 2 Pro. This analysis demonstrates that the protein epitopes needed to elicit a neutralizing immune response to SARS-CoV-2 are correctly folded and accessible.

We also generated VLPS displaying multiple copies of a second prefusion-stabilized variant of the $S$ protein, called HexaPro, which was reported by Hsieh et al. to be more stable than S-2P and give a higher expression yield ${ }^{19}$. We expressed a variant of HexaPro containing a C-terminal AviTag and a his-tag, which we termed S $_{6 \text { Pro }}$ (Figure 3a). VLP-S 6 Pro were generated and characterized (Figure 3) as described above for VLP$\mathrm{S}_{2 \mathrm{Pro}}$

\section{Protective efficacy and antibody response to a single immunization in Syrian hamsters}

We next evaluated the antibody responses elicited by these nanoparticle-based vaccine candidates in Syrian hamsters. $\mathrm{We}^{3}$ and others ${ }^{20}$ have demonstrated that Syrian hamsters are highly susceptible to SARS-CoV-2 infection and present with pathological phenotypes similar to those of infected humans, making hamsters an ideal animal model to evaluate vaccine candidates. Hamsters ( 4 groups; 3 animals/group) were immunized with VLP-S $_{2 \text { Pro, }}$ VLP-S 6 Pro, MS2-SA VLPs alone, or PBS along with Alhydrogel, an aluminum hydroxide base adjuvant. The hamsters were bled 28 days after immunization to characterize their antibody responses (Figure 4a). Hamsters immunized once with the VLP-S conjugates had appreciable levels of IgG antibodies against the RBD of the $S$ protein as determined by ELISA, with end-point titers ranging from $2 \times 10^{4}$ to $8 \times 10^{4}$ and high neutralizing antibody titers ranging from 320-640 (Table 1). In contrast, as expected, negligible anti-S antibodies were detected in hamsters immunized with the controls (VLPs alone or PBS). 
Four weeks after immunization, the animals were intranasally inoculated with $10^{3}$ plaque-forming units of SARS-CoV-2/UT-NCGM02/Human/2020/Tokyo3. While the animals in both control groups experienced

significant weight loss, those immunized with VLP-S 2 Pro had recovered their average initial weight by day 3 , and those immunized with VLP-S 6 Pro showed a slight increase in body weight over this 3-day period (Figure 4b).

Three days after virus challenge, which is when virus levels in the lungs peak ${ }^{3}$, the animals were sacrificed and lung and nasal turbinate samples were collected. As expected, animals in both control groups (PBS and MS2-SA VLPs) had high viral loads in the lungs; however, in hamsters immunized with VLP-S Pro $_{\text {Or }}$ VLP-S 6 Pro no infectious virus was detected in the lungs (Figure 4c). The lack of infectious virus in the lungs was consistent with the differences observed in body weight change between the vaccine and control groups. Moreover, despite the intranasal mode of challenge with SARS-CoV-2, the hamsters immunized with VLP-S 2 ro or VLP-S 6 Pro had less virus in their nasal turbinates (Figure 4d), with mean titers more than 150-fold lower (VLP$\left.\mathrm{S}_{2 \mathrm{Pro}}\right)$ and more than 700 -fold lower $\left(\mathrm{VLP}_{\mathrm{S}} \mathrm{S}_{6 \mathrm{Pro}}\right)$ relative to MS2-SA VLP controls.

In summation, we have developed a highly effective nanoparticle-based vaccine that provides protection in an animal model against SARS-CoV-2 after a single immunization. The development of multiple effective vaccine platforms is critical because vaccines remain the best approach for protection from current and future pandemics. Given factors such as manufacturing costs, the number of people that must be immunized, and societal habits, an ideal vaccine against SARS-CoV-2 would offer protection after only one immunization. The nanoparticle-based vaccine platform described here should be broadly applicable for protecting against important pathogens including, but not limited to, SARS-CoV-2 and influenza viruses.

\section{Methods}

\section{Expression and Purification of MS2}

DNA encoding single chain MS2 coat protein dimer with an AviTag inserted between the fourteenth and fifteenth residues of the second coat protein monomer was cloned into pET-28b between the Ndel and Xhol restrictions sites by GenScript Biotech Corporation (Piscataway, NJ). The MS2 dimer with the inserted AviTag was cotransformed with pAcm-BirA (Avidity LLC) into BL21(DE3) competent E. coli (New England BioLabs) according 
1 to the manufacturer's instructions. The transformation was added to $5 \mathrm{~mL}$ of $2 x Y T$ media and grown overnight

2 at $37^{\circ} \mathrm{C}$. The $5-\mathrm{mL}$ starter culture was then added to $1 \mathrm{~L}$ of $2 x Y T$ media, which was incubated shaking at $37^{\circ} \mathrm{C}$ 3 until induction with IPTG (1M; GoldBio) at an OD of 0.6. Immediately after induction, biotin (50 $\mu \mathrm{M})$ was added 4 to the culture and the incubator temperature was reduced to $30^{\circ} \mathrm{C}$. After overnight incubation, the culture was 5 centrifuged for 7 minutes at 7,000xg and the supernatant was decanted. The cell pellet was homogenized into

$25 \mathrm{~mL}$ of $20 \mathrm{mM}$ Tris Base ( $\mathrm{pH}$ 8.0) supplemented with lysozyme (0.5 mg/mL; Alfa Aesar), a protease inhibitor tablet (Sigma-Aldrich), and benzonase (125 units; EMD Millipore). The resuspended cells were then kept on ice and stirred intermittently for 20 minutes. Sodium deoxycholate (Alfa Aesar) was then added to a final concentration of $0.1 \%(\mathrm{w} / \mathrm{v})$, and the mixture was sonicated for 3 minutes at $35 \%$ amplitude with a pulse of 3 seconds on and 3 seconds off (Sonifier S-450, Branson Ultrasonics). The sonication was repeated after allowing the lysate to cool on ice for two minutes. Next, the lysed cells were centrifuged for 30 minutes at $27,000 \mathrm{xg}$. The supernatant was collected, and centrifuged again for 15 minutes at $12,000 \mathrm{xg}$. The resulting supernatant was then diluted 3-fold in $20 \mathrm{mM}$ Tris Base and filtered with a $0.45-\mu \mathrm{m}$ bottle-top filter (VWR). Then, $25 \mathrm{~mL}$ of the diluted lysate was loaded onto four HiScreen Capto Core 700 columns (Cytiva) in series using an AKTA start system. The columns were washed with approximately 3 column volumes of $20 \mathrm{mM}$ Tris Base while fractions were collected. Fractions were subsequently analyzed for purity and recovery of MS2 by using SDS-PAGE. Desirable fractions were pooled, concentrated by using a $10 \mathrm{kDa}$ MWCO centrifugal filter (Millipore Sigma), and further purified by using a Superdex 200 Increase 10/300 column (Cytiva). MS2 was quantified by using a bicinchoninic acid assay (BCA) (Thermo Scientific).

\section{Expression, Refolding, and Purification of Streptavidin (SA)}

SA was expressed, refolded, and purified essentially as previously described ${ }^{21,22}$. Briefly, DNA encoding SA (Addgene plasmid \#46367, a gift from Mark Howarth) ${ }^{21}$ was transformed into BL21(DE3) cells according to the manufacturer's protocol. The transformation was split among four culture tubes each containing $5 \mathrm{~mL}$ of $2 \mathrm{xYT}$ media, which were incubated overnight at $37^{\circ} \mathrm{C}$. Each $5 \mathrm{~mL}$ culture was added to one of four $1 \mathrm{~L}$ flasks of $2 \times Y T$ and grown at $37^{\circ} \mathrm{C}$. Upon reaching an OD of 0.6 , expression of inclusion bodies was induced using IPTG (1 M; GoldBio) and the temperature of the incubator was reduced to $30^{\circ} \mathrm{C}$. After incubation overnight, the culture was centrifuged for 7 minutes at $7000 \mathrm{xg}$ such that 4 liters of culture resulted in two cell pellets. Each pellet was 
1 resuspended in $50 \mathrm{~mL}$ of resuspension buffer (50 mM Tris, $100 \mathrm{mM} \mathrm{NaCl}, \mathrm{pH}$ 8.0) supplemented with lysozyme

2 (1 mg/mL; Alfa Aesar) and benzonase (500 units; EMD Millipore) and was allowed to incubate at $4^{\circ} \mathrm{C}$ for $1 \mathrm{~h}$ with

occasional mixing. These mixtures were then homogenized, brought to a concentration of $0.1 \%$ (w/v) sodium deoxycholate (Alfa Aesar), and sonicated (Sonifier S-450, Branson Ultrasonics) for 3 minutes at 35\% amplitude with a pulse of 3 seconds on and 3 seconds off. The resulting lysate was then centrifuged for 15 minutes at $27,000 \times g$. The supernatant was discarded, and the two pellets were each again resuspended in $50 \mathrm{~mL}$ of resuspension buffer supplemented with lysozyme $(1 \mathrm{mg} / \mathrm{mL}$; Alfa Aesar) and the lysis procedure was repeated. This procedure resulted in two inclusion body pellets, which were then washed. Each inclusion body pellet was resuspended in $50 \mathrm{~mL}$ of wash buffer \#1 (50 mM Tris, $100 \mathrm{mM} \mathrm{NaCl,} 100 \mathrm{mM}$ EDTA, 0.5\% (v/v) Triton X-100, $\mathrm{pH} 8.0$ ), homogenized, and sonicated for 30 seconds at an amplitude of $35 \%$. Each mixture was then centrifuged at $27,000 \mathrm{xg}$ for 15 minutes and the supernatant was discarded. This wash was repeated twice. The two inclusion body pellets resulting from the third round of the initial wash were each resuspended in $50 \mathrm{~mL}$ of wash buffer \#2 (50 mM Tris, 10 mM EDTA, pH 8.0), homogenized, and sonicated for 30 seconds at an amplitude of $35 \%$. Each mixture was then centrifuged at $15,000 x g$ for 15 minutes. This wash was repeated once. The two resulting washed inclusion body pellets were then completely unfolded by resuspension in $10 \mathrm{~mL}$ of a $7.12 \mathrm{M}$ guanidine hydrochloride solution. This mixture was stirred at room temperature for $1 \mathrm{~h}$, and subsequently centrifuged at $12,000 x g$ for 10 minutes. The supernatant was drawn into a syringe, which was loaded onto a syringe pump, and added at a rate of $30 \mathrm{~mL} / \mathrm{h}$ to $1 \mathrm{~L}$ of chilled PBS that was being stirred rapidly. This solution of refolded protein was stirred continuously overnight at $4^{\circ} \mathrm{C}$. Insoluble protein was then pelleted by centrifugation at 7,000xg for 15 minutes and discarded. The supernatant containing the folded SA was filtered by using a $0.45-$ $\mu \mathrm{m}$ bottle-top filter. The resulting filtrate was stirred vigorously, and ammonium sulfate was slowly added to a concentration of $1.9 \mathrm{M}$ to precipitate out protein impurities. After being stirred for $3 \mathrm{~h}$ at $4{ }^{\circ} \mathrm{C}$, the precipitate was removed by centrifugation for 10 minutes at 7,000xg. The supernatant was then filtered by using a $0.45-\mu \mathrm{m}$ bottle-top filter. The ammonium sulfate concentration of the resulting filtrate was brought up to a total concentration of $3.68 \mathrm{M}$ and stirred for $3 \mathrm{~h}$ at $4^{\circ} \mathrm{C}$ to precipitate the SA. The SA precipitate was pelleted by centrifugation at 7,000xg for 20 minutes, and resuspended in $20 \mathrm{~mL}$ of Iminobiotin Affinity Chromatography (IBAC) binding buffer (50 mM Sodium Borate, $300 \mathrm{mM} \mathrm{NaCl}, \mathrm{pH} 11.0$ ). This SA solution was then passed 
1 through $5 \mathrm{~mL}$ of Pierce Iminobiotin Agarose (Thermo Scientific) in a gravity flow column (G-Biosciences) that

2 had been pre-equilibrated with 5 column volumes of IBAC binding buffer. The IBAC column containing the bound

3 SA was then washed with 20 column volumes of IBAC binding buffer. Then, 8 column volumes of elution buffer

4 were passed through the column. The eluate was collected, dialyzed into PBS, and concentrated using a 10

$5 \mathrm{kDa}$ MWCO centrifugal filter (Millipore Sigma). SA was quantified by measuring the UV absorption at $280 \mathrm{~nm}$.

Assembly and Purification of MS2-SA VLPS

7 Biotinylated MS2 was added dropwise to a molar excess of concentrated SA solution that was stirred vigorously

8 in a 5-mL glass vial. After a 30-minute incubation, the MS2-SA VLP was separated from the excess SA through

9 SEC with a Superdex 200 Increase 10/300 column (Cytiva). The MS2-SA VLP was quantified by boiling a small 10 aliquot at $90^{\circ} \mathrm{C}$ in Nu-PAGE lithium dodecyl sulfate (LDS) sample buffer (Invitrogen) for 30 minutes and running

11 the sample on a polyacrylamide gel. SA standards with known concentrations quantified by UV absorption at $12280 \mathrm{~nm}$ were also run on the gel. Comparing the intensities of the bands resulting from the SA standards with 13 the intensity of the band representing the SA from the MS2-SA allowed for quantification of the VLP.

Expression and Purification of SARS-CoV-2 S proteins

DNA encoding the S-2P $\mathrm{P}^{14}$ and HexaPro ${ }^{19}$ prefusion-stabilized versions of the SARS-CoV-2 S ectodomain (residues 1-1208) with a C-terminal T4 fibritin trimerization motif, AviTag, and a his-tag were cloned into pcDNA3.1 between the Ncol and Xhol restriction sites by Gene Universal Inc. (Newark, DE). These plasmids were transfected into Expi293F cells using the ExpiFectamine Transfection Kit and protocol (Thermo Fisher Scientific). Five days after transfection, the cells were pelleted by centrifugation for 20 minutes at $5500 \mathrm{xg}$. The supernatant was dialyzed into PBS and passed through $1 \mathrm{~mL}$ of HisPur Ni-NTA resin (Thermo Fisher Scientific) in a gravity flow column (G-Biosciences). The column was then washed with $40 \mathrm{~mL}$ of wash buffer (42 mM sodium bicarbonate, $8 \mathrm{mM}$ sodium carbonate, $300 \mathrm{mM} \mathrm{NaCl}, 20 \mathrm{mM}$ imidazole). The S proteins were eluted from the column by incubating the Ni-NTA resin with $3 \mathrm{~mL}$ of elution buffer $(42 \mathrm{mM}$ sodium bicarbonate, $8 \mathrm{mM}$ sodium carbonate, $300 \mathrm{mM} \mathrm{NaCl}, 300 \mathrm{mM}$ imidazole) for 5 minutes before allowing for flow by gravity. This elution procedure was repeated twice, resulting in $9 \mathrm{~mL}$ of eluate. The eluate was concentrated by using a 10- 
1 kDa MWCO centrifugal filter (Millipore Sigma). S proteins were buffer exchanged into $20 \mathrm{mM} \mathrm{Tris,} 20 \mathrm{mM} \mathrm{NaCl}$,

$2 \mathrm{pH} 8.0$ to allow for in vitro biotinylation and were quantified by using the BCA assay (Thermo Scientific).

3 In vitro biotinylation of AviTagged MS2 and SARS-CoV-2 S

4 Biotinylation was performed in vitro using a BirA biotin-protein ligase standard reaction kit (Avidity) following the 5 manufacturer's protocol. In brief, the protein solution (either MS2 or SARS-CoV-2 S) was buffer exchanged into 6 a $20 \mathrm{mM}$ Tris, $20 \mathrm{mM} \mathrm{NaCl}, \mathrm{pH} 8.0$ buffer and the protein concentration was adjusted to $45 \mu \mathrm{M}$. BirA and a 7 proprietary mixture containing biotin, ATP, and magnesium acetate (Biomix B) was added to the protein solution. 8 This solution was shaken vigorously at $37^{\circ} \mathrm{C}$. After $2 \mathrm{~h}$ at $37^{\circ} \mathrm{C}$, more Biomix $\mathrm{B}$ was added, and the solution was 9 nutated at $4^{\circ} \mathrm{C}$ overnight. The proteins of interest were then purified through SEC with a Superdex 200 Increase 10/300 column (Cytiva). Biotinylated S proteins were quantified by using the BCA assay (Thermo Scientific).

\section{Expression and Purification of CR3022 and ACE2-FC}

The variable regions of the heavy and light chains of CR3022 $2^{18}$ were cloned into the TGEX-HC and TGEX-LC vectors (Antibody Design Labs), respectively, according to the manufacturer's protocol. Likewise, ACE2 (residues 1-615) was cloned into TGEX-HC. The DNA was then transfected into Expi293F cells by using the ExpiFectamine Transfection Kit (Thermo Fisher Scientific) following the provided protocol, and the cells were incubated in a humidified incubator at $37^{\circ} \mathrm{C}$ and $8 \% \mathrm{CO}_{2}$ for 5 days. The cells were then centrifuged at $5500 \times \mathrm{g}$ for 20 minutes. The supernatant media was diluted 2-fold in PBS and run through a 1-mL MabSelect SuRe column (Cytiva) according to the manufacturer's operation manual to purify the proteins. CR3022 and ACE2-Fc were quantified by using the BCA assay (Thermo Scientific).

\section{SDS-PAGE}

Protein samples were diluted 4-fold in Nu-PAGE lithium dodecyl sulfate (LDS) sample buffer (Invitrogen). The samples were then boiled at $90^{\circ} \mathrm{C}$ for 30 minutes. PageRuler Plus Prestained Protein Ladder (Thermo Scientific) and protein samples were pipetted into the wells of a $4 \%-12 \%$ Bis-Tris gel (Invitrogen), which was run in MESSDS buffer at $4^{\circ} \mathrm{C}$ for $1 \mathrm{~h}$ at $110 \mathrm{~V}$. The gel was stained with SimplyBlue SafeStain (Invitrogen) and subsequently de-stained. Once sufficiently de-stained, the gel was imaged by using the ChemiDoc MP imaging system (BioRad). 
Preparation of VLP-S

MS2-SA and biotinylated S protein were mixed in a stoichiometric ratiofound by using analytical SEC. We used analytical SEC to characterize mixtures consisting of $5 \mu \mathrm{g}$ of biotinylated S protein and varying amounts of MS2SA VLP. The ratio of the mixture that contained the least MS2-SA VLP and also did not have excess S protein appear on the chromatogram was the stoichiometric ratio used to create the VLP-S. The concentration of the VLP-S was adjusted such that the solution contained $0.12 \mu \mathrm{g}$ of $S$ per $\mu \mathrm{L}$. The VLP-S were further characterized by use of ELISA, SEC, and DLS as described below.

\section{Characterization of S and VLP-S by ELISA}

VLP-S and S protein in PBS were coated onto a Nunc Maxisorp 96-well plate such that each well contained 0.1 $\mu \mathrm{g}$ of $\mathrm{S}$ protein in $100 \mu \mathrm{L}$. After $1 \mathrm{~h}$, the protein solutions were discarded from the wells and each well was blocked with $200 \mu \mathrm{L}$ of $5 \%$ BSA (EMD Millipore) in PBST (PBS with $0.05 \%$ Tween-20) for 45 minutes. The plate was then washed twice with PBST, and CR3022 and ACE2-Fc in 1\% BSA in PBST were added to the appropriate wells such that each well contained either one CR3022 or ACE2-Fc molecule per S trimer. One hour later, the wells were washed twice with PBST and a horseradish peroxidase-conjugated anti-human IgG Fc fragment antibody (MP Biomedicals) in 1\% BSA in PBST was added to each well and left to incubate for $1 \mathrm{~h}$. Then, the plate was washed twice with PBST and developed with TMB substrate solution (Thermo Scientific) for 3 minutes; the reaction was then stopped with $0.16 \mathrm{M}$ sulfuric acid. The absorbance of each well at $450 \mathrm{~nm}$ was read using a Spectramax i3x plate reader (Molecular Devices).

\section{Analytical SEC}

A Superdex 200 Increase 10/300 column (Cytiva) was equilibrated with PBS. The 1-mL sample loop was washed with PBS and then $950 \mu \mathrm{l}$ of either VLP-S solution or S alone was loaded into the sample loop. Each sample included $5 \mu \mathrm{g}$ of $S$ protein. The sample loop was then flushed with PBS such that the sample was directed through the column at a flowrate of $0.5 \mathrm{~mL} / \mathrm{min}$. One column volume of PBS was run through the column. Unicorn 7 (Cytiva) was used to control the system and to output a chromatogram of UV absorbance at $210 \mathrm{~nm}$.

\section{Dynamic Light Scattering}


1 A UVette (Eppendorf) containing $100 \mu \mathrm{L}$ of VLP-S at a concentration of approximately $0.05 \mu \mathrm{S}$ per $\mu \mathrm{L}$ was

2 loaded into a DynaPro NanoStar Dynamic Light Scattering detector (Wyatt Technology). For each measurement,

3 Dynamics software (Wyatt Technology) was used to allow the temperature to equilibrate to $25^{\circ} \mathrm{C}$, to collect 10

4 acquisitions, and to output the results. Results were displayed by \% Mass using the Isotropic Spheres model.

$5 \quad$ Virus and Titration Assays

6 The virus isolate SARS-CoV-2/UT-NCGM02/Human/2020/Tokyo was used in this study and was previously $7 \quad$ characterized in Syrian hamsters ${ }^{3}$.

8 Virus titrations were performed on Vero E6/TMPRSS2 cells that were obtained from the National Institute of

9 Infectious Diseases, Japan ${ }^{23}$. Cells were maintained in Dulbecco's modified Eagle's medium (DMEM) containing $1010 \%$ fetal bovine serum (FBS) and antibiotic/antimycotic solution along with G418 (1 mg/ml).

11 To determine virus titers, confluent Vero E6/TMPRSS2 cells were infected with $100 \mu$ of undiluted or 10-fold dilutions $\left(10^{-1}\right.$ to $\left.10^{-5}\right)$ of clarified lung or nasal turbinate homogenates. After a 30 -minute incubation, the inoculum was removed, the cells were washed once, and then overlaid with $1 \%$ methylcellulose solution in DMEM with $5 \%$ FBS. The plates were incubated for three days, and then the cells were fixed and stained with $20 \%$ methanol 15 and crystal violet to count the plaques.

Hamster Immunization Study

17 The immunization study in hamsters was performed after approval by the Institutional Animal Care and Use 18 Committee at the University of Wisconsin. Golden Syrian hamsters (4-week-old females) were immunized with 19 either $60 \mu \mathrm{g}$ of SARS-CoV-2 S protein presented on the MS2-SA VLP, an equal amount of MS2-SA VLP without 20 the S protein, or an equal volume of sterile phosphate-buffered saline (PBS) by subcutaneous inoculation. 21 Alhydrogel ( $2 \%$ solution; InvivoGen) added at an equal volume was thoroughly mixed with each vaccine 22 preparation before inoculation. Animals were infected by intranasal inoculation with $10^{3}$ plaque-forming units 23 (PFU) of SARS-CoV-2 while under isoflurane anesthesia. Animals were monitored daily for signs of illness and 24 their body weights were recorded daily. Three days after infection, the animals were humanely sacrificed and 25 lung tissue and nasal turbinate samples were collected. 
1 Serum was isolated from blood samples collected via the sublingual vein before the immunization and challenge

2 with virus.

3 Detection of Antibodies Against the RBD of SARS-CoV-2 S in Immunized Hamsters by ELISA

4 The ELISA was performed using a recombinant SASR-CoV-2 S RBD protein produced in Expi293F cells 5 (Thermo Fisher Scientific) and then C-terminal his-tag purified by using TALON metal affinity resin. ELISA plates 6 were coated overnight at $4^{\circ} \mathrm{C}$ with $50 \mu \mathrm{l}$ of the RBD protein at a concentration of $2 \mu \mathrm{g} / \mathrm{ml}$ in PBS. After being 7 blocked with PBS containing $0.1 \%$ Tween 20 (PBS-T) and 3\% milk powder, the plates with incubated in duplicate 8 with heat-inactivated serum diluted in PBS-T with 1\% milk powder. Anti-hamster lgG secondary antibody 9 conjugated with horseradish peroxidase (Invitrogen; 1:7,000 dilution) was used for detection. Plates were developed with SigmaFast o-phenylenediamine dihydrochloride solution (Sigma), and the reaction was stopped with the addition of $3 \mathrm{M}$ hydrochloric acid. The absorbance was measured at a wavelength of $490 \mathrm{~nm}\left(\mathrm{OD}_{490}\right)$. Background absorbance measurements from serum collected before immunization were subtracted from the absorbance measurements from plasma collected before challenge for each dilution. IgG antibody endpoint titers were defined as the highest plasma dilution with an $\mathrm{OD}_{490}$ cut-off value of 0.15 .

Neutralization Assay

Virus ( 100 PFU) was incubated with the same volume of two-fold dilutions of heat-inactivated serum for 30 minutes at $37^{\circ} \mathrm{C}$. The antibody/virus mixture was added to confluent Vero E6/TMPRSS2 cells that were plated at 30,000 cells per well the day prior in 96 -well plates. The cells were incubated for 3 days at $37^{\circ} \mathrm{C}$ and then fixed and stained with $20 \%$ methanol and crystal violet solution. Virus neutralization titers were determined as the reciprocal of the highest serum dilution that completely prevented cytopathic effects.

Biosafety Statement

Research with SARS-CoV-2 was approved by the University of Wisconsin-Madison's Institutional Biosafety Committee and performed under biosafety level 3 agriculture (BSL-3Ag) containment at the Influenza Research Institute, University of Wisconsin-Madison. The laboratory is approved for such use by the Centers for Disease Control and Prevention. The BSL-3Ag facility used was designed to exceed the standards outlined in Biosafety in Microbiological and Biomedical Laboratories (5th edition). 


\section{Data availability}

The data needed to support the conclusions of this study have been included in the paper. The data that support the plots within the paper are available from the corresponding authors upon reasonable request.

\section{Acknowledgements}

Addgene plasmid \#46367 (pET21-Streptavidin-Glutamate_Tag) was a gift from Mark Howarth. RSK acknowledges support from the Garry Betty/ V Foundation Chair Fund as well as seed funding from the School of Chemical \& Biomolecular Engineering at the Georgia Institute of Technology. YK acknowledges support from the Collaborative Influenza Vaccine Innovation Centers (CIVIC, contract 75N93019C00051) and the Center for Research on Influenza Pathogenesis (CRIP, HHSN272201400008C) funded by the National Institutes of Allergy and Infectious Diseases.

\section{Author contributions}

All authors were involved in designing the experiments. S.J.F., S.C, P.J.H., M.K., and T.M. performed the experiments and analyzed the data. S.J.F., P.J.H., and R.S.K. wrote the manuscript.

\section{Competing interests}

The authors declare no competing interests.

\section{References}

1 Frietze, K. M., Peabody, D. S. \& Chackerian, B. Engineering virus-like particles as vaccine platforms. Curr Opin Virol 18, 44-49, doi:10.1016/j.coviro.2016.03.001 (2016).

2 Valegard, K., Murray, J. B., Stockley, P. G., Stonehouse, N. J. \& Liljas, L. Crystal structure of an RNA bacteriophage coat protein-operator complex. Nature 371, 623-626, doi:10.1038/371623a0 (1994).

3 Imai, M. et al. Syrian hamsters as a small animal model for SARS-CoV-2 infection and countermeasure development. Proc Natl Acad Sci U S A 117, 16587-16595, doi:10.1073/pnas.2009799117 (2020).

4 Krammer, F. SARS-CoV-2 vaccines in development. Nature 586, 516-527, doi:10.1038/s41586-0202798-3 (2020).

5 Yang, J. et al. Molecular interaction and inhibition of SARS-CoV-2 binding to the ACE2 receptor. Nat Commun 11, 4541, doi:10.1038/s41467-020-18319-6 (2020).

6 Addetia, A. et al. Neutralizing Antibodies Correlate with Protection from SARS-CoV-2 in Humans during a Fishery Vessel Outbreak with a High Attack Rate. J Clin Microbio/ 58, doi:10.1128/JCM.02107-20 (2020).

7 Walls, A. C. et al. Structure, Function, and Antigenicity of the SARS-CoV-2 Spike Glycoprotein. Cell 181, 281-292 e286, doi:10.1016/j.cell.2020.02.058 (2020).

8 Shin, M. D. et al. COVID-19 vaccine development and a potential nanomaterial path forward. Nat Nanotechnol 15, 646-655, doi:10.1038/s41565-020-0737-y (2020).

9 Bachmann, M. F. \& Jennings, G. T. Vaccine delivery: a matter of size, geometry, kinetics and molecular patterns. Nat Rev Immunol 10, 787-796, doi:10.1038/nri2868 (2010). 
110 Plummer, E. M. \& Manchester, M. Viral nanoparticles and virus-like particles: platforms for contemporary vaccine design. Wiley Interdiscip Rev Nanomed Nanobiotechnol 3, 174-196, doi:10.1002/wnan.119 (2011).

11 Bachmann, M. F. \& Zinkernagel, R. M. Neutralizing antiviral B cell responses. Annu Rev Immunol 15, 235-270, doi:10.1146/annurev.immunol.15.1.235 (1997).

12 Zhang, B. et al. A platform incorporating trimeric antigens into self-assembling nanoparticles reveals SARS-CoV-2-spike nanoparticles to elicit substantially higher neutralizing responses than spike alone. Sci Rep 10, 18149, doi:10.1038/s41598-020-74949-2 (2020).

13 Peabody, D. S. et al. Immunogenic display of diverse peptides on virus-like particles of RNA phage MS2. J Mol Biol 380, 252-263, doi:10.1016/j.jmb.2008.04.049 (2008).

14 Wrapp, D. et al. Cryo-EM structure of the 2019-nCoV spike in the prefusion conformation. Science 367, 1260-1263, doi:10.1126/science.abb2507 (2020).

15 Lan, J. et al. Structure of the SARS-CoV-2 spike receptor-binding domain bound to the ACE2 receptor. Nature 581, 215-220, doi:10.1038/s41586-020-2180-5 (2020).

$16 \mathrm{Ju}$, B. et al. Human neutralizing antibodies elicited by SARS-CoV-2 infection. Nature 584, 115-119, doi:10.1038/s41586-020-2380-z (2020).

17 Shi, R. et al. A human neutralizing antibody targets the receptor-binding site of SARS-CoV-2. Nature 584, 120-124, doi:10.1038/s41586-020-2381-y (2020).

18 ter Meulen, J. et al. Human monoclonal antibody combination against SARS coronavirus: synergy and coverage of escape mutants. PLoS Med 3, e237, doi:10.1371/journal.pmed.0030237 (2006).

19 Hsieh, C. L. et al. Structure-based design of prefusion-stabilized SARS-CoV-2 spikes. Science 369, 15011505, doi:10.1126/science.abd0826 (2020).

20 Sia, S. F. et al. Pathogenesis and transmission of SARS-CoV-2 in golden hamsters. Nature 583, 834-838, doi:10.1038/s41586-020-2342-5 (2020).

21 Fairhead, M., Krndija, D., Lowe, E. D. \& Howarth, M. Plug-and-play pairing via defined divalent streptavidins. J Mol Biol 426, 199-214, doi:10.1016/j.jmb.2013.09.016 (2014).

22 Howarth, M. \& Ting, A. Y. Imaging proteins in live mammalian cells with biotin ligase and monovalent streptavidin. Nat Protoc 3, 534-545, doi:10.1038/nprot.2008.20 (2008).

23 Matsuyama, S. et al. Enhanced isolation of SARS-CoV-2 by TMPRSS2-expressing cells. Proc Natl Acad Sci U S A 117, 7001-7003, doi:10.1073/pnas.2002589117 (2020). 
2 Table 1. Antibody responses to immunization in Syrian hamsters.

\begin{tabular}{|l|c|c|c|}
\hline $\begin{array}{l}\text { Vaccine } \\
\text { Group }\end{array}$ & Animal \# & $\begin{array}{c}\text { RBD IgG } \\
\text { Endpoint Titer* }\end{array}$ & $\begin{array}{c}\text { Neutralizing } \\
\text { Antibody Titer }^{\dagger}\end{array}$ \\
\hline PBS & 1 & $<10$ & $<10$ \\
\hline & 2 & $<10$ & $<10$ \\
\hline & 3 & $<10$ & $<10$ \\
\hline $\begin{array}{l}\text { MS2-SA } \\
\text { VLP }\end{array}$ & 1 & $<10$ & $<10$ \\
\hline & 2 & $<10$ & $<10$ \\
\hline & 3 & $<10$ & $<10$ \\
\hline VLP-S & & 20,480 & 320 \\
\hline & 1 & 81,920 & 640 \\
\hline & 2 & 20,480 & 320 \\
\hline VLP-S S & 3 & 81,920 & 640 \\
\hline & 1 & 81,920 & 640 \\
\hline & 3 & 40,960 & 320 \\
\hline
\end{tabular}

*Viral antibody endpoint titers against the RBD (receptor-binding domain) expressed as the reciprocal of the highest dilution with an optical density at $490 \mathrm{~nm}$ (OD490) cutoff value $>0.15$; sera were collected on day 28 after immunization.

†Viral neutralization titers in sera collected on day 28 after immunization. 
a
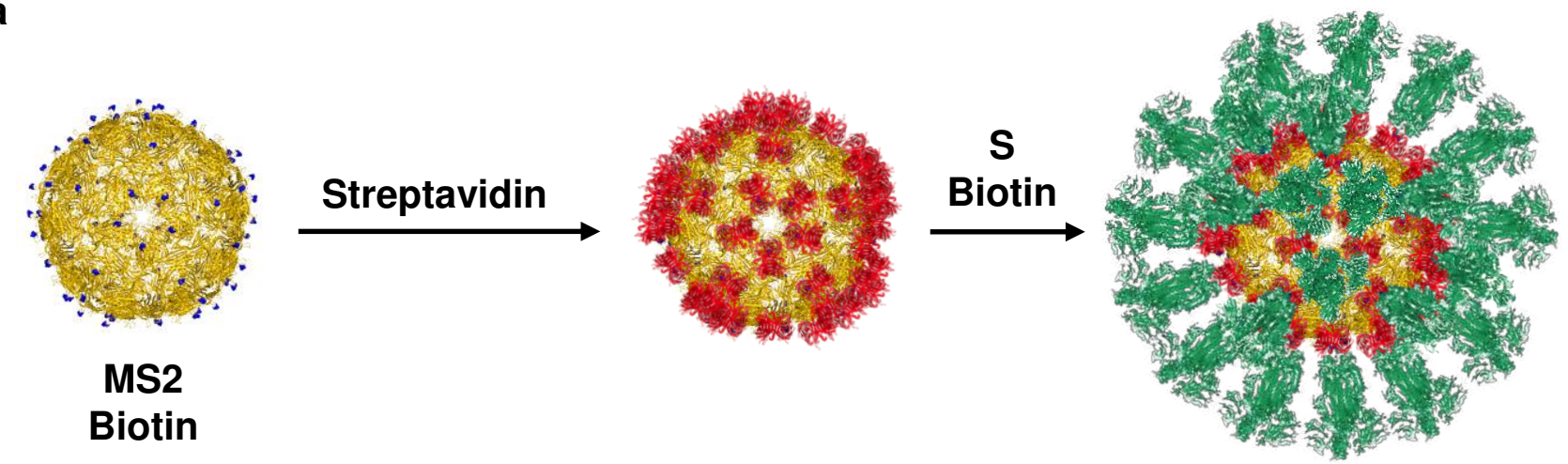

b

C
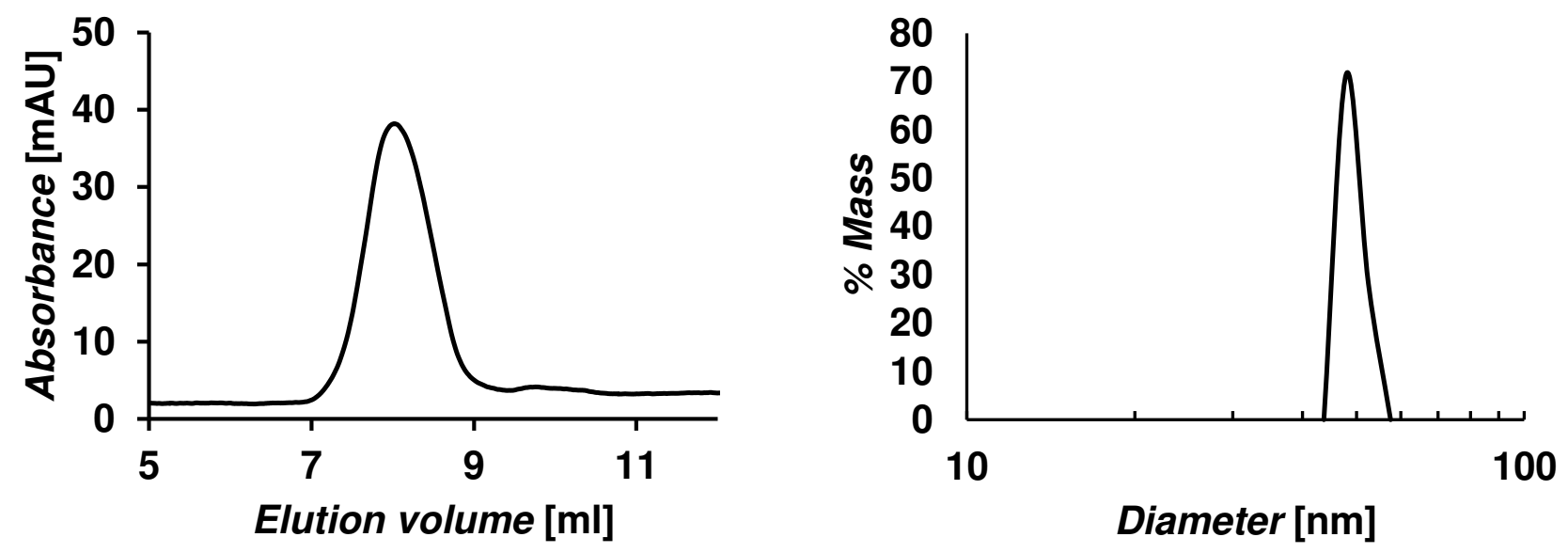

Figure 1. Assembly of VLP-S and characterization of MS2-SA VLP. a) Scheme illustrating assembly of VLPS, where biotinylated MS2 (yellow, PDB: 2MS2) is added to streptavidin (red, PDB:3RY2) to create the VLP. $\mathrm{S}$ (green, PDB: 6VSB) biotinylated at the C-terminus is mixed with the VLP to create VLP-S. Biotinylated residues are colored blue. b) Size exclusion chromatography trace for MS2-SA VLP. c) Characterization of the MS2-SA VLP by dynamic light scattering. 
a

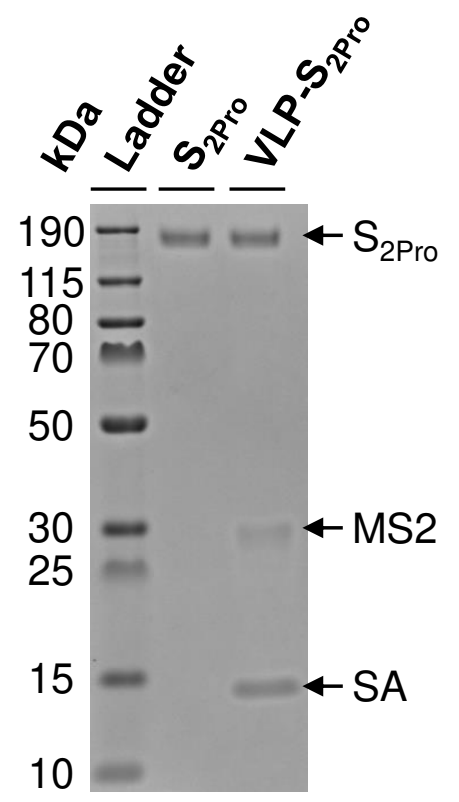

C

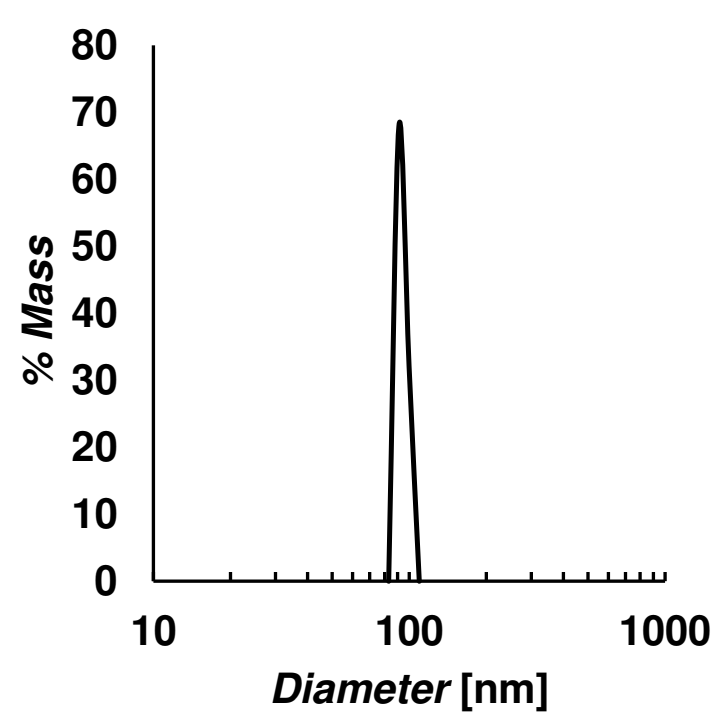

b

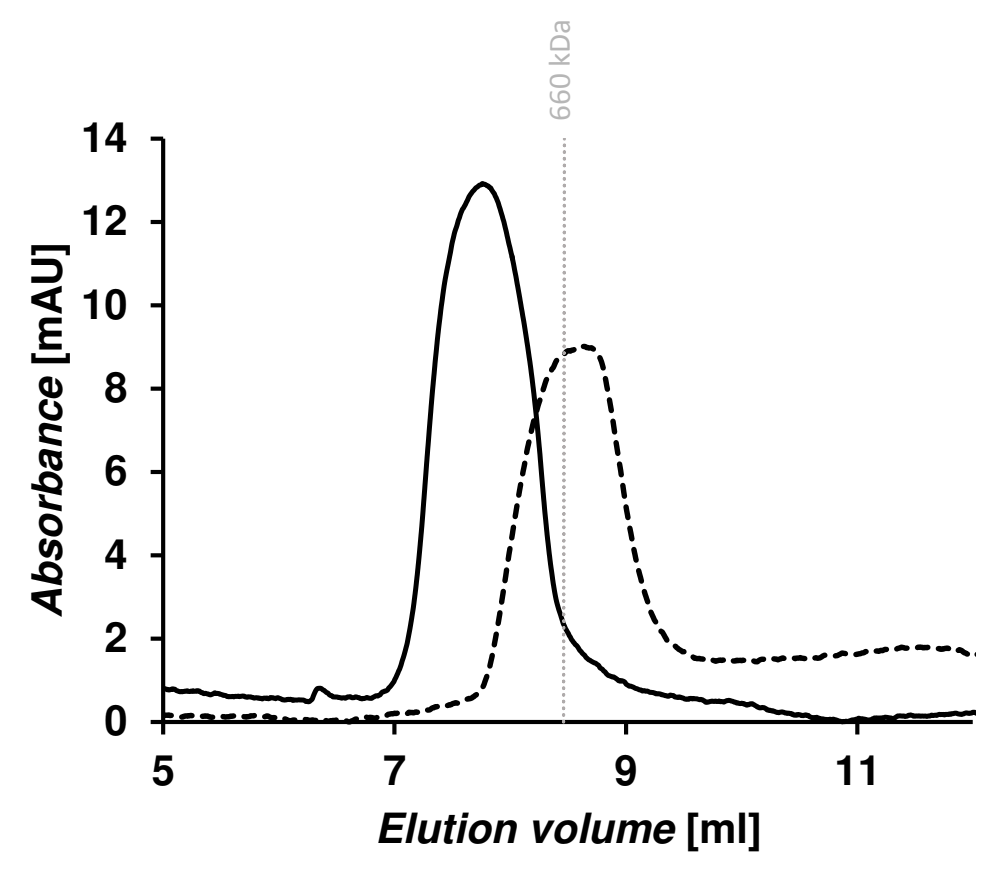

d

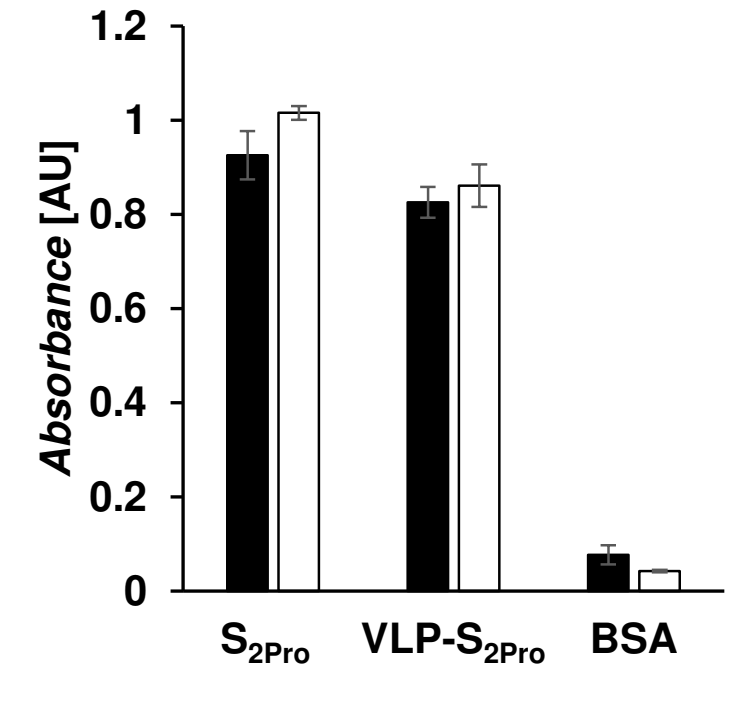

Figure 2. Characterization of $S_{2 \text { Pro }}$ and VLP-S $S_{2 P r o}$ a) SDS-PAGE characterization of $S_{2 \text { Pro }}$ and VLP- $S_{2 \text { Pro }}$. The VLP- $\mathrm{S}_{2 \text { Pro }}$ has been boiled to disrupt the streptavidin-biotin conjugation. b) Size exclusion chromatography traces for $\mathrm{S}_{2 \mathrm{Pro}}$ (dashed line) and VLP- $\mathrm{S}_{2 \text { Pro }}$ (solid line). The vertical gray line represents the peak elution volume of the molecular weight standard thyroglobulin $(660 \mathrm{kDa})$. c) Characterization of the VLP- $\mathrm{S}_{2 \text { Pro }}$ (solid line) by dynamic light scattering. d) Characterization of the binding of Fc-ACE2 (black) and CR3022 (white) to $\mathrm{S}_{2 \text { Pro }}$ and VLP-S ${ }_{2 \text { Pro }}$ by ELISA (mean $\pm \mathrm{SD}, \mathrm{n}=6$ ). 
a

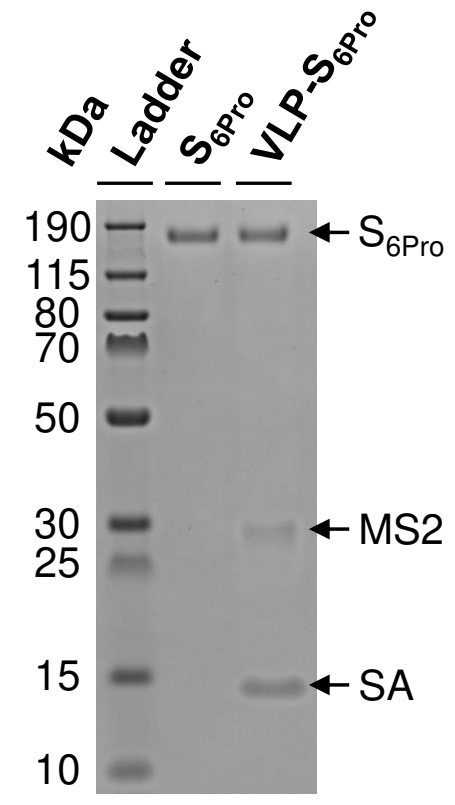

C

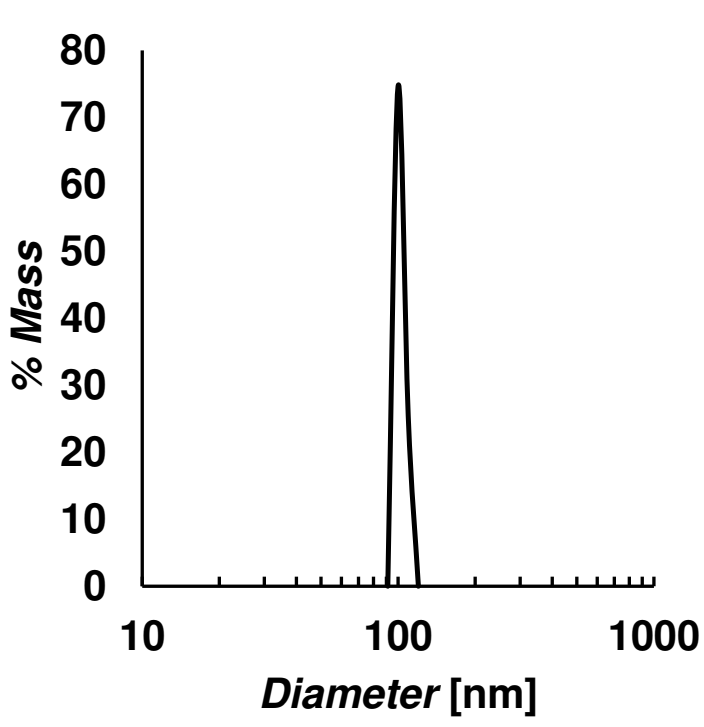

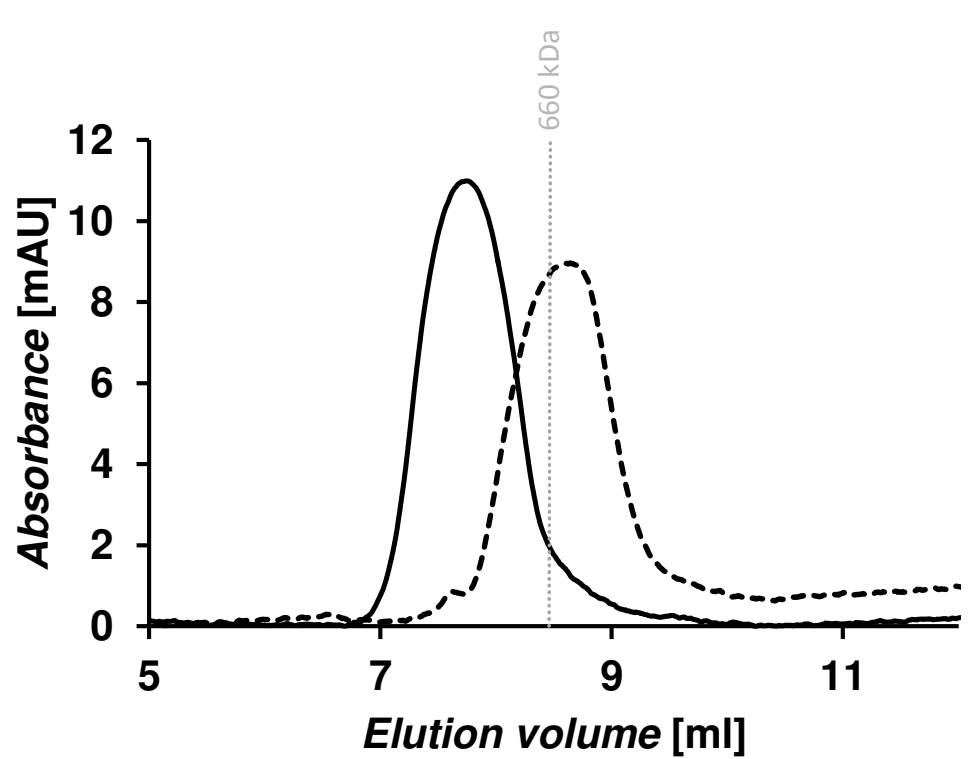

d

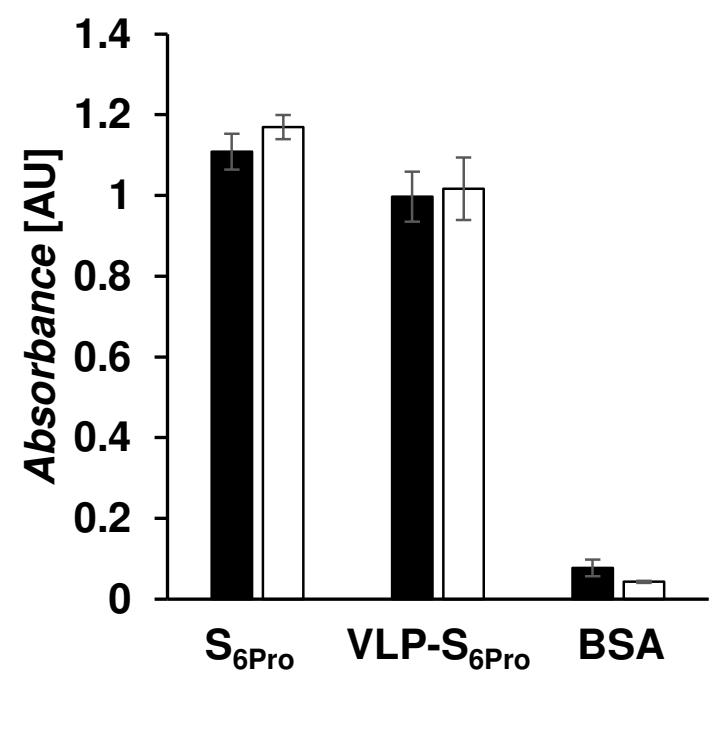

Figure 3. Characterization of $\mathrm{S}_{6 \text { Pro }}$ and VLP-S $\mathrm{S}_{6 \text { Pro }}$ a) SDS-PAGE characterization of $\mathrm{S}_{6 \text { Pro }}$ and VLP- $\mathrm{S}_{6 \text { Pro }}$. The VLP- $S_{6 \text { Pro }}$ has been boiled to disrupt the streptavidin-biotin conjugation. b) Size exclusion chromatography traces for $\mathrm{S}_{6 \mathrm{Pro}}$ (dashed line) and VLP- $\mathrm{S}_{6 \text { Pro }}$ (solid line). The vertical gray line represents the peak elution volume of the molecular weight standard thyroglobulin $(660 \mathrm{kDa})$. c) Characterization of the VLP- $\mathrm{S}_{6 \text { Pro }}$ (solid line) by dynamic light scattering. d) Characterization of the binding of Fc-ACE2 (black) and CR3022 (white) to $\mathrm{S}_{6 \text { Pro }}$ and VLP-S $\mathrm{S}_{6 \text { Pro }}$ by ELISA (mean $\pm \mathrm{SD}, \mathrm{n}=6$ ). 


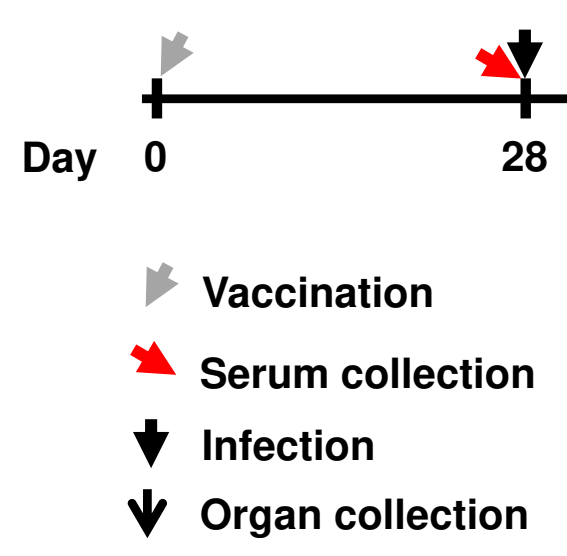

C

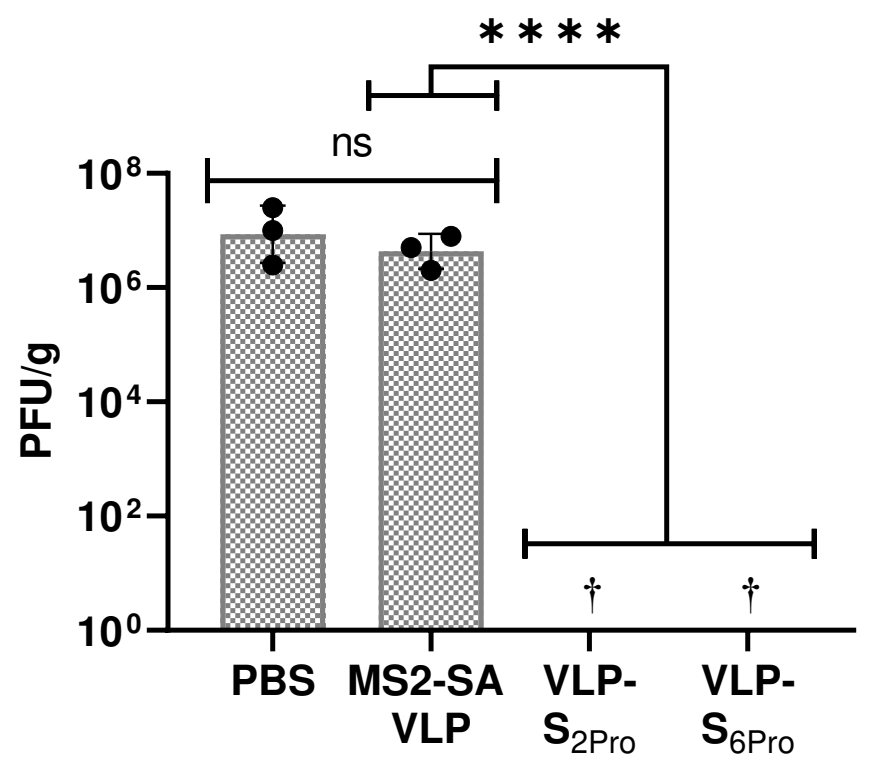

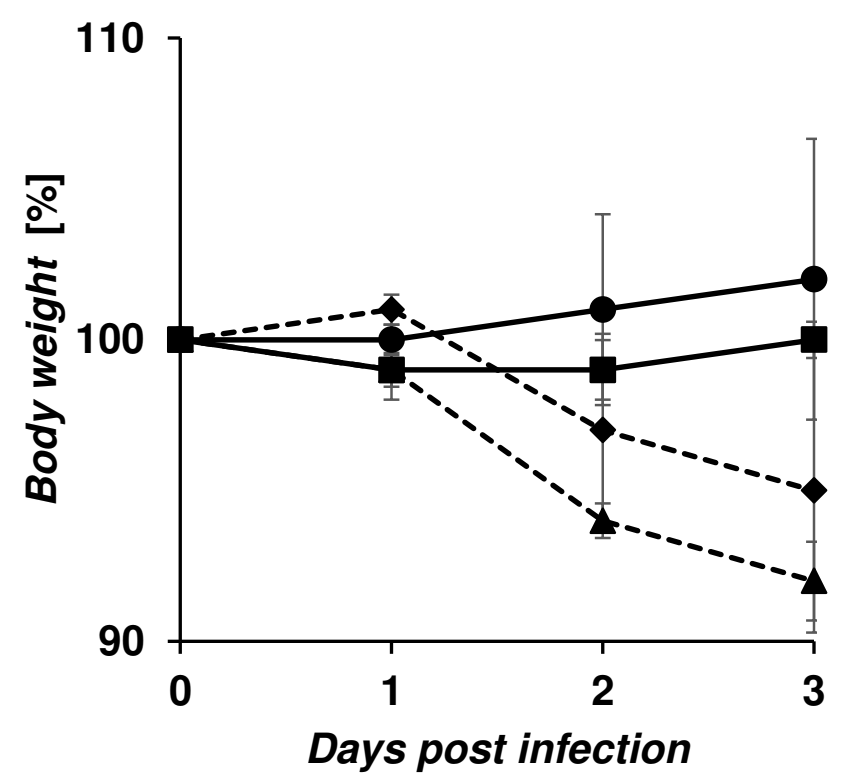

d

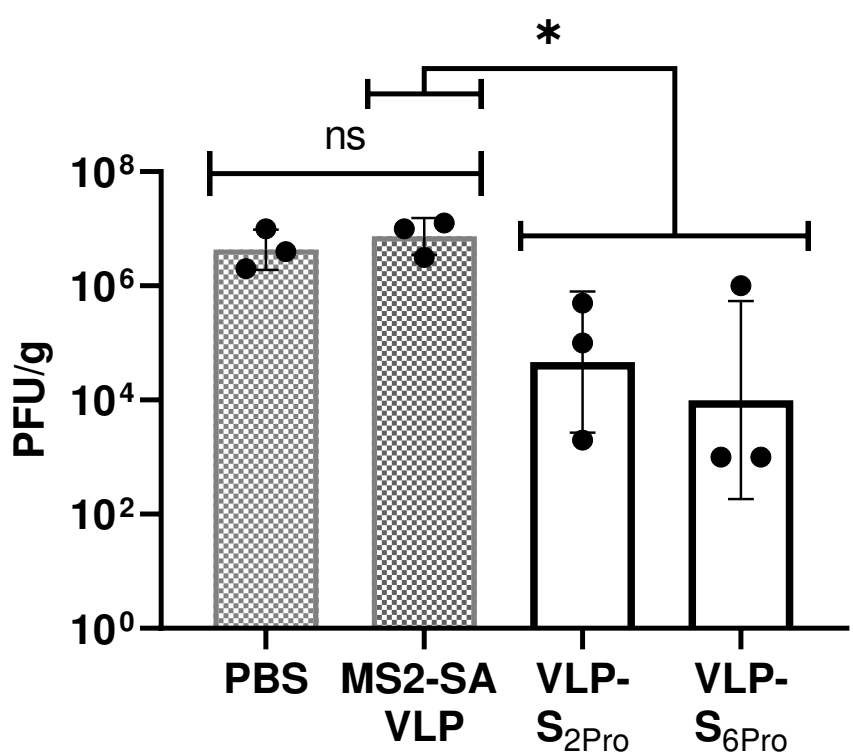

Figure 4. Protective efficacy of VLP-S. a) Schedule for vaccination of hamsters, serum collection, infection, and organ collection. b) Body weight of hamsters immunized with a single dose of either VLP-

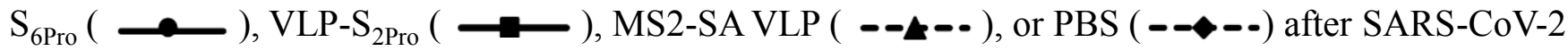
infection (mean $\pm \mathrm{SD}, \mathrm{n}=3$ ). c) Viral titer in the lungs of hamsters immunized with either PBS, MS2-SA VLP, VLP-S ${ }_{2 \text { Pro }}$ or VLP-S SPro $_{6}$ three days after SARS-CoV-2 infection (geometric mean with geometric $\mathrm{SD}, \mathrm{n}=3) . \dagger-$ No infectious virus was detected in the lungs of hamsters immunized with VLP-S $\mathrm{S}_{2 \mathrm{Pro}}$ or VLP-S $_{6 \text { Pro }}$ (detection limit $10 \mathrm{PFU} / \mathrm{g}$ ). ns: not statistically significant, $* * * * p<0.0001$, determined by a one-way analysis of variance (ANOVA) and Dunnett post-hoc multiple comparison between groups $(\alpha=$ 0.1 ). Assumptions of the normality of residuals and homogeneity of variance were validated by the Shapiro-Wilk test and the Brown-Forsythe test, respectively. d) Viral titer in the nasal turbinates of hamsters immunized with either PBS, MS2-SA VLP, VLP-S ${ }_{2 \text { Pro }}$ or VLP-S ${ }_{6 \text { Pro }}$ three days after SARS-CoV2 infection (geometric mean with geometric $\mathrm{SD}, \mathrm{n}=3$ ). ns: not statistically significant, $* \mathrm{p}<0.1$, determined by a one-way analysis of variance (ANOVA) and Dunnett post-hoc multiple comparison between groups $(\alpha=0.1)$. Assumptions of the normality of residuals and homogeneity of variance were validated by the Shapiro-Wilk test and the Brown-Forsythe test, respectively. 
Figures

a

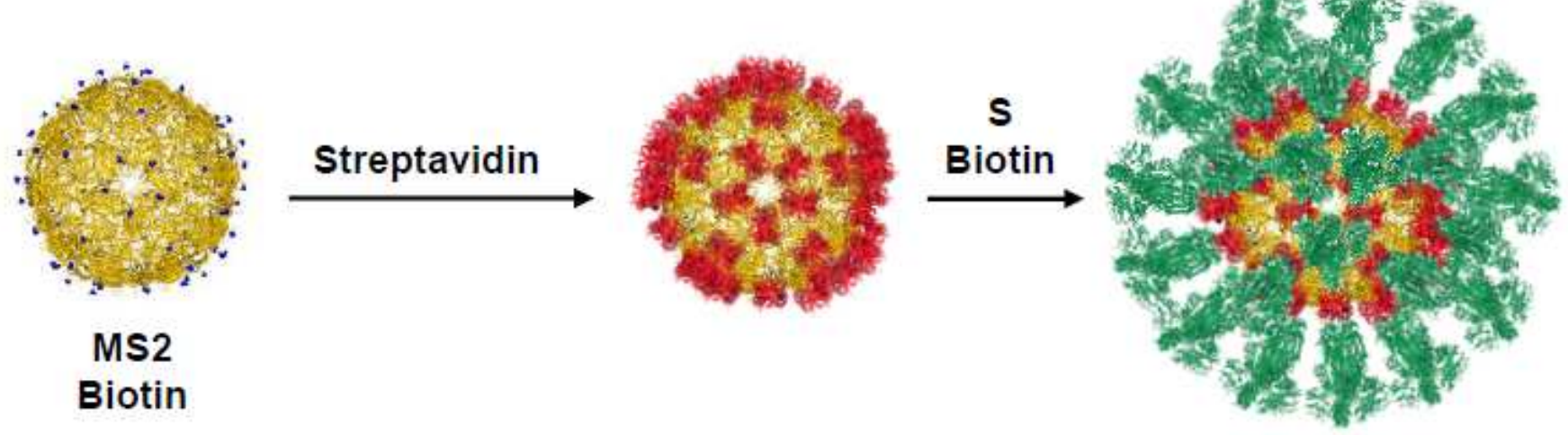

b

C
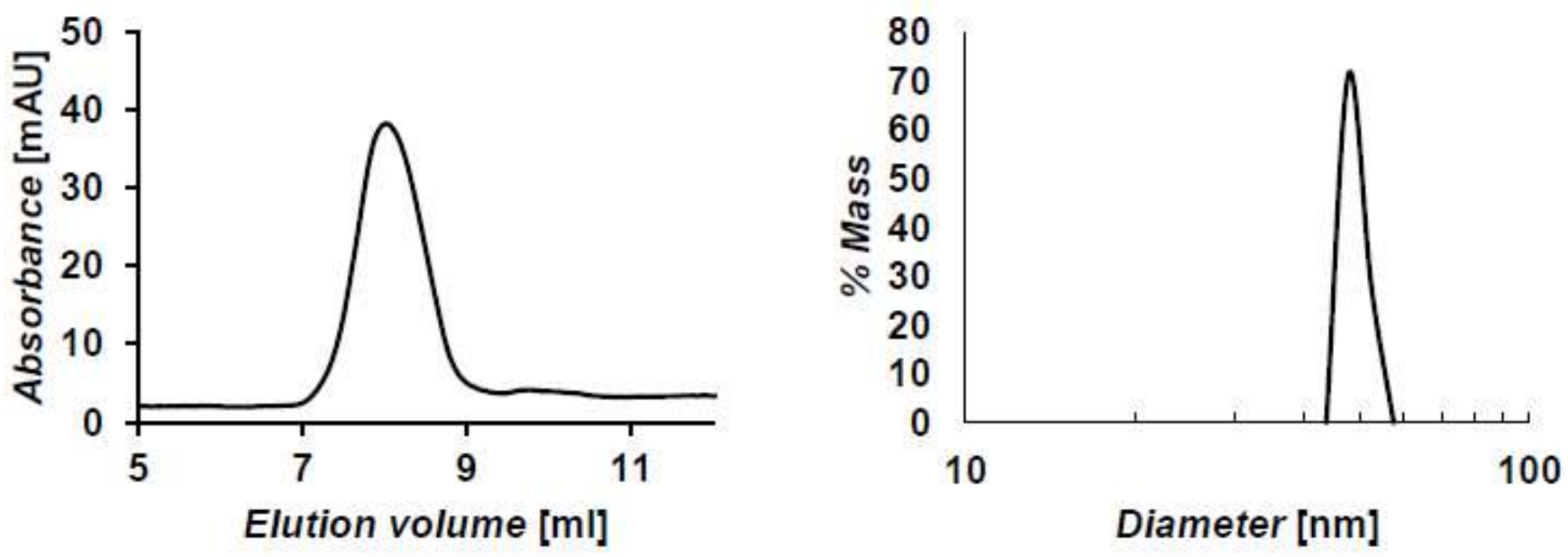

Figure 1

Assembly of VLP-S and characterization of MS2-SA VLP. a) Scheme illustrating assembly of VLP-S, where biotinylated MS2 (yellow, PDB: 2MS2) is added to streptavidin (red, PDB:3RY2) to create the VLP. S (green, PDB: 6VSB) biotinylated at the C-terminus is mixed withthe VLP to create VLP-S. Biotinylated residues are colored blue. b) Size exclusion chromatography trace for MS2-SA VLP. c) Characterization of the MS2-SA VLP by dynamic light scattering. 
a

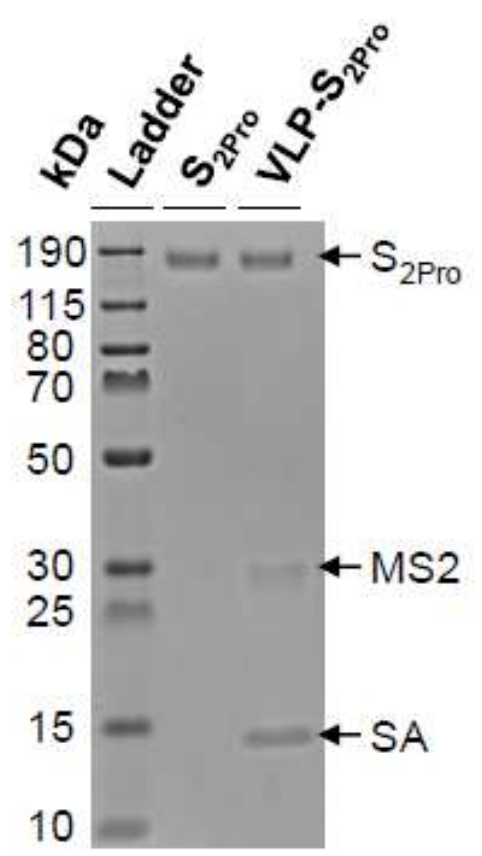

C

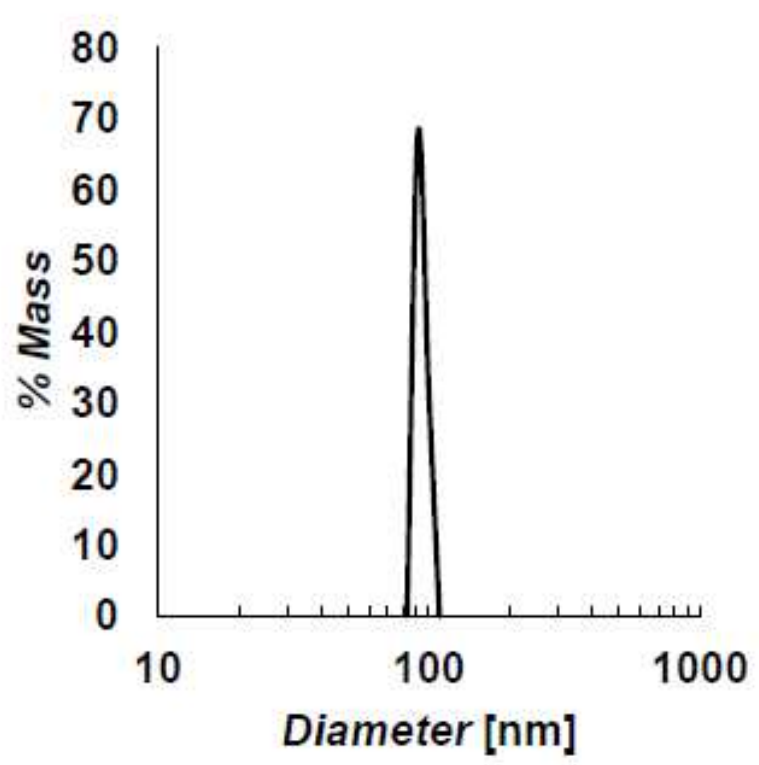

b

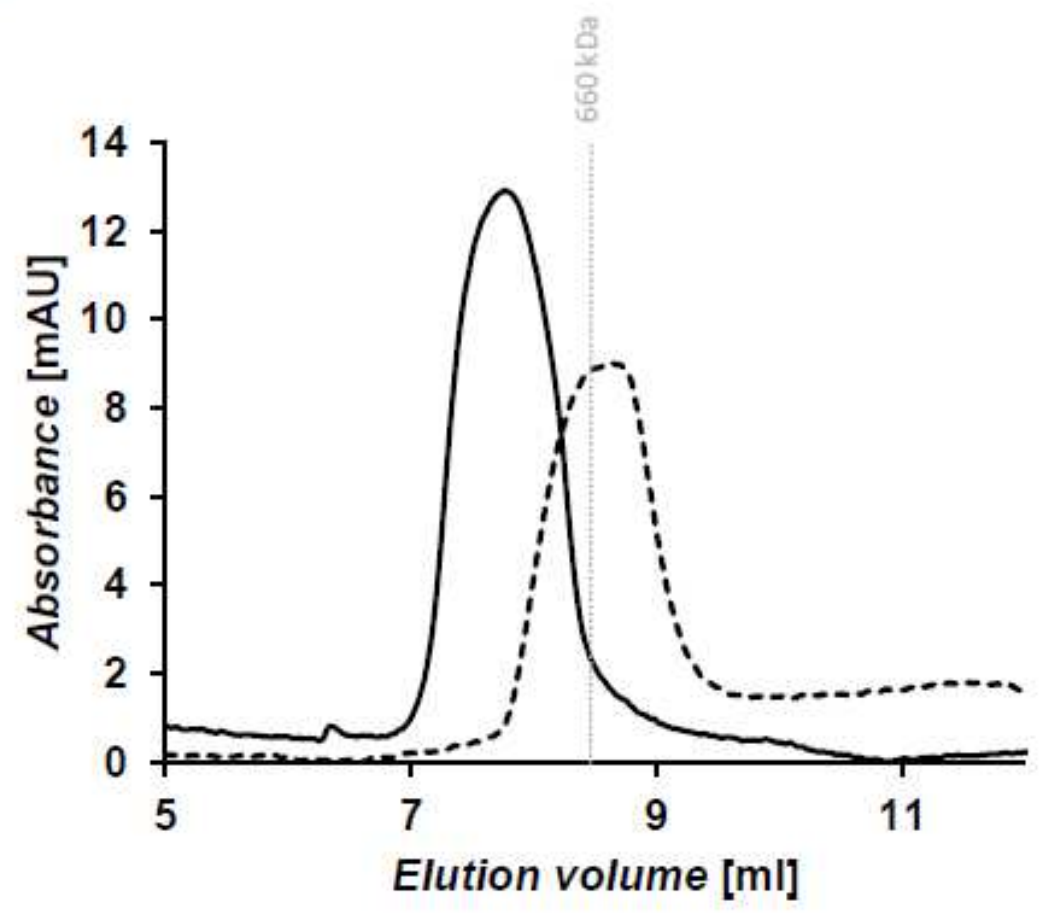

d

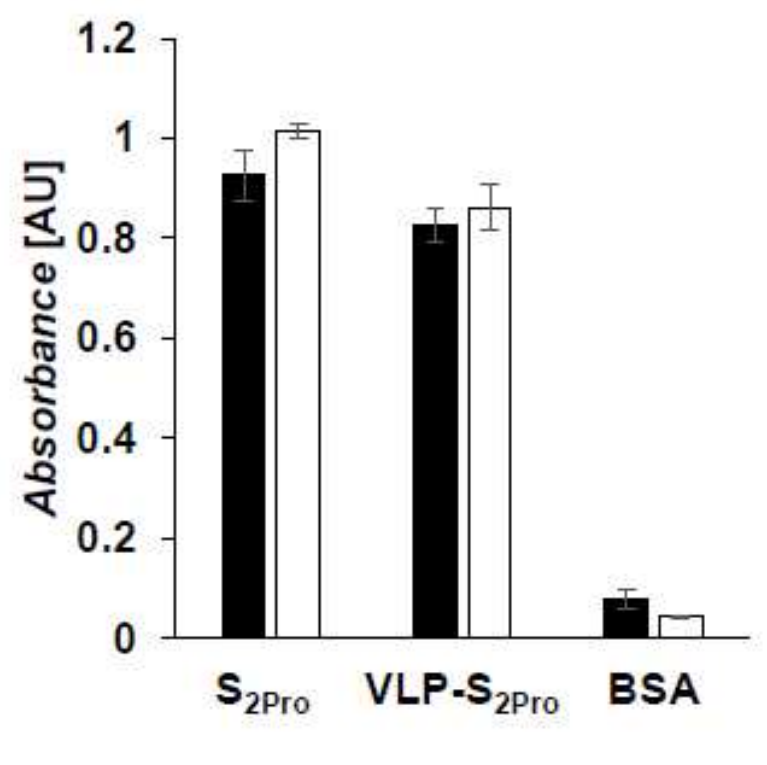

Figure 2

Characterization of S2Proand VLP-S2Pro. a) SDS-PAGE characterization of S2Proand VLP-S2Pro. The VLP-S2Prohas been boiled to disrupt the streptavidin-biotin conjugation. b) Size exclusion chromatography traces for S2Pro(dashed line) and VLP-S2Pro(solid line). The vertical gray line represents the peak elution volume of the molecular weight standard thyroglobulin (660 kDa). c) 
Characterization of the VLP-S2Pro(solid line) by dynamic light scattering. d) Characterization of the binding of Fc-ACE2 (black) and CR3022 (white) to S2Proand VLP-S2Proby ELISA (mean $\pm S D, n=6$ ).

a

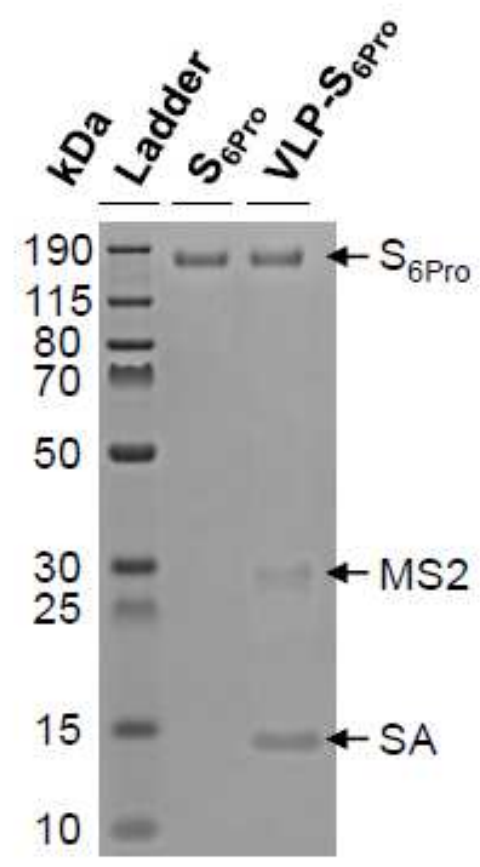

C

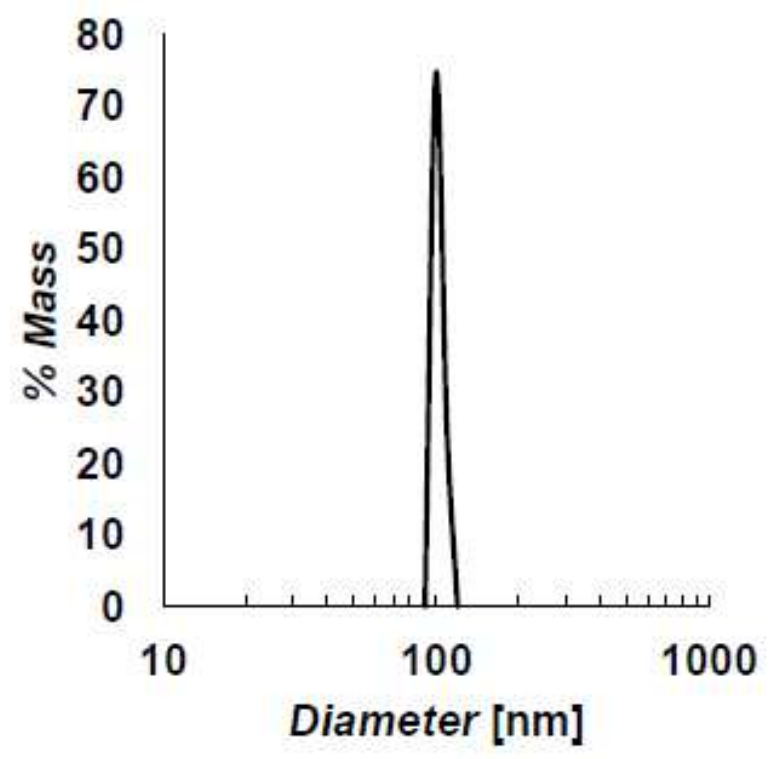

b

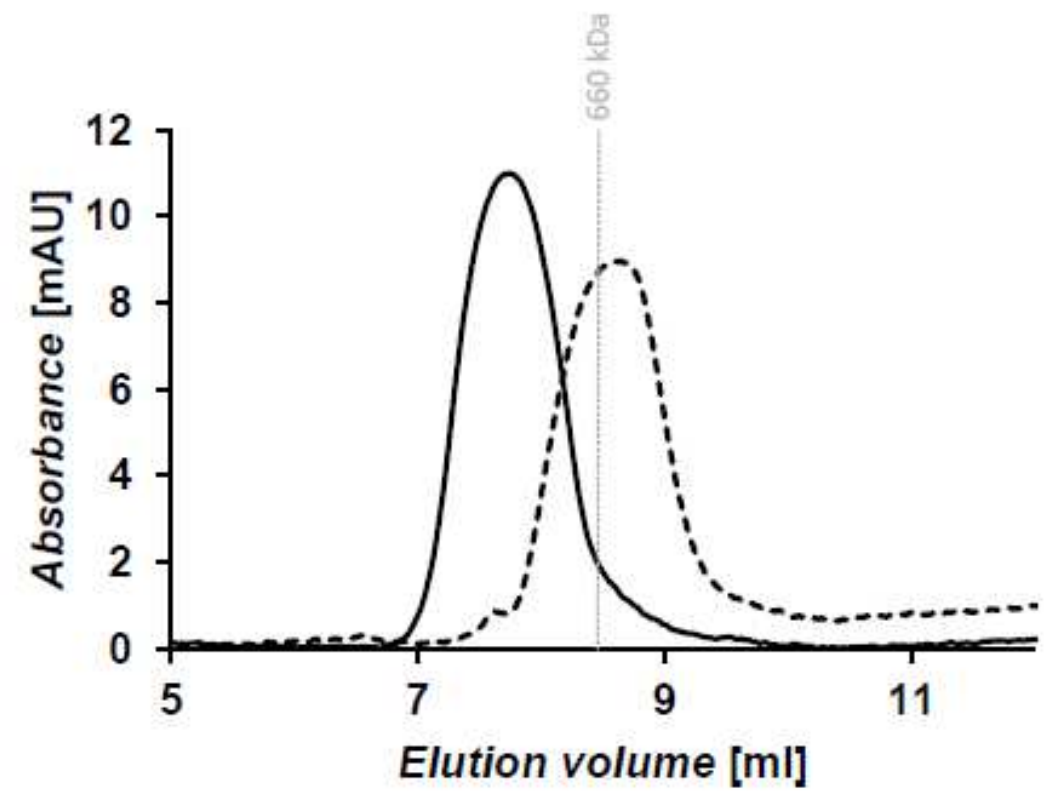

d

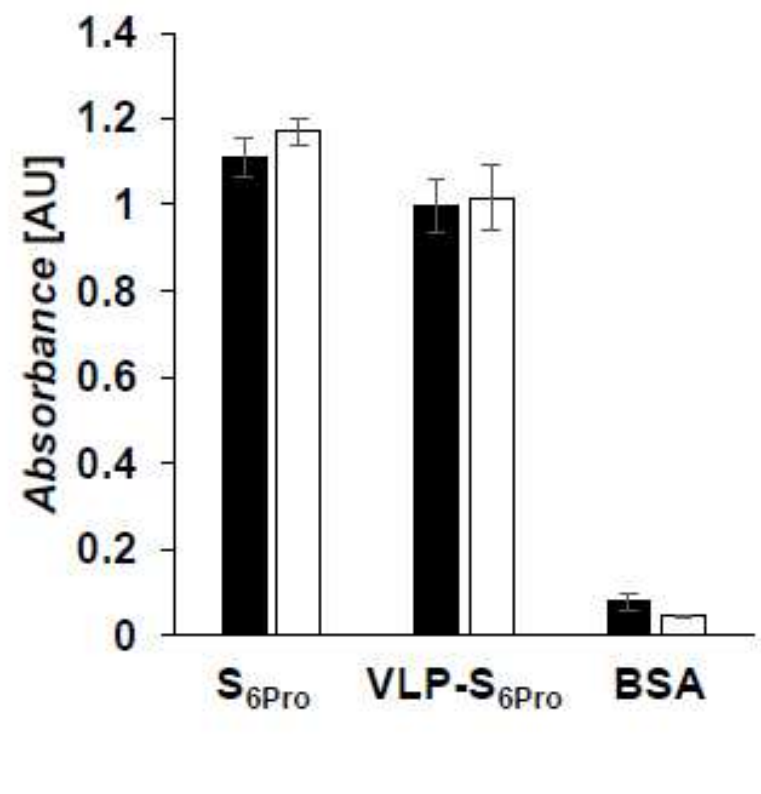

Figure 3

Characterization of S6Proand VLP-S6Pro. a) SDS-PAGE characterization of S6Proand VLP-S6Pro. The VLP-S6Prohas been boiled to disrupt the streptavidin-biotin conjugation. b) Size exclusion chromatography traces for S6Pro(dashed line) and VLP-S6Pro(solid line). The vertical gray line 
represents the peak elution volume of the molecular weight standard thyroglobulin (660 kDa). c) Characterization of the VLP-S6Pro(solid line) by dynamic light scattering. d) Characterization of the binding of Fc-ACE2 (black) and CR3022 (white) to S6Proand VLP-S6Pro by ELISA (mean $\pm S D, n=6$ ).

a

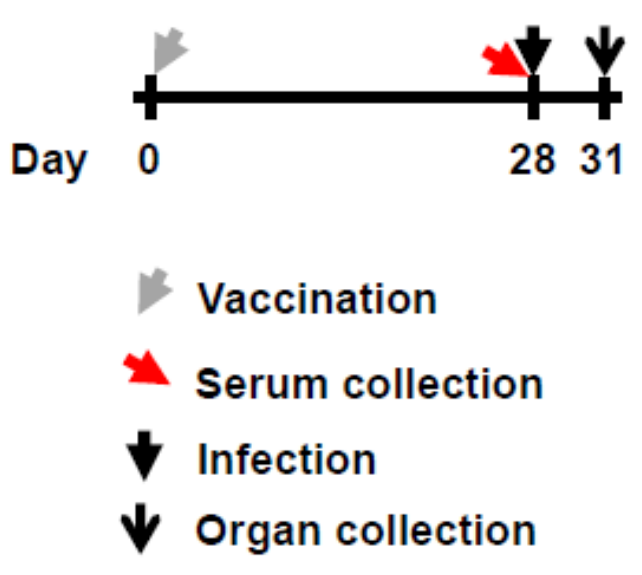

C

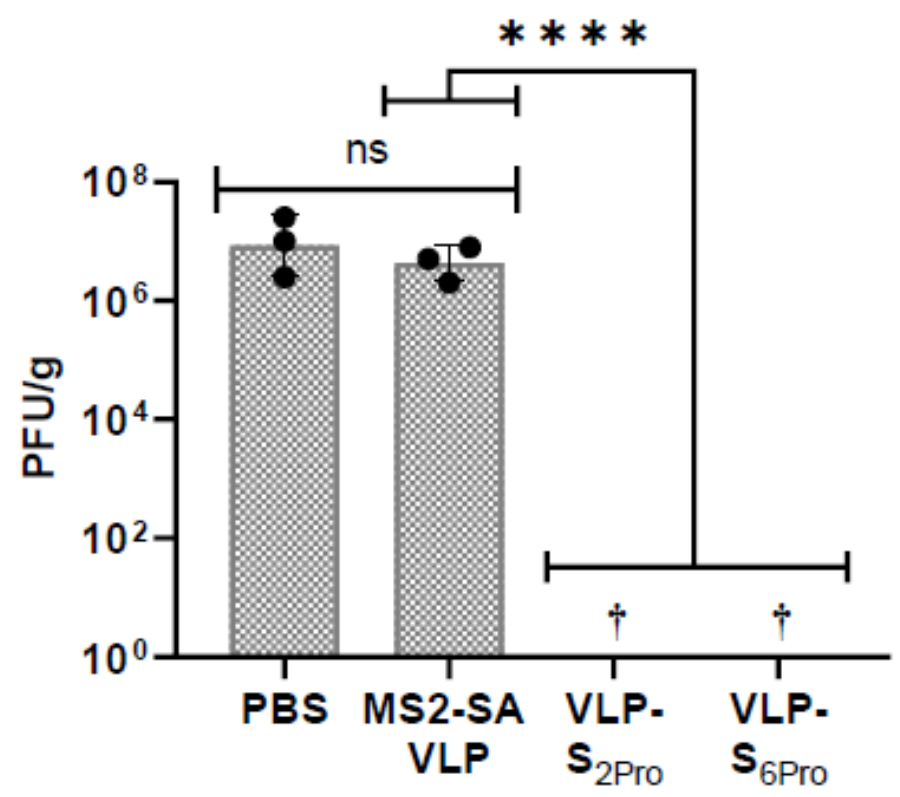

b

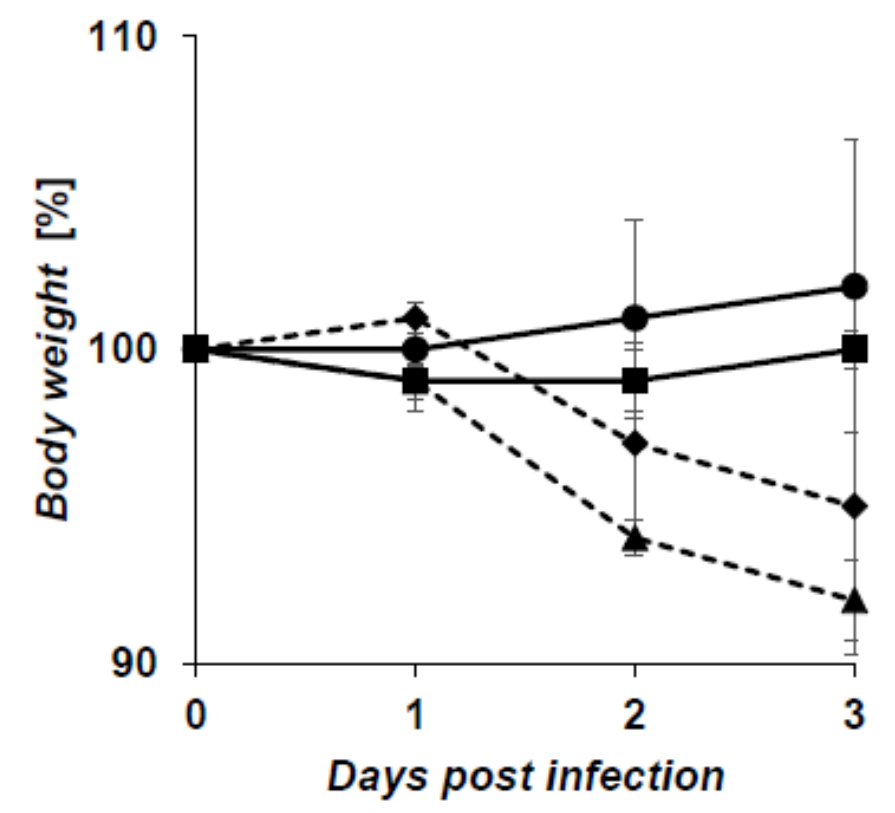

d

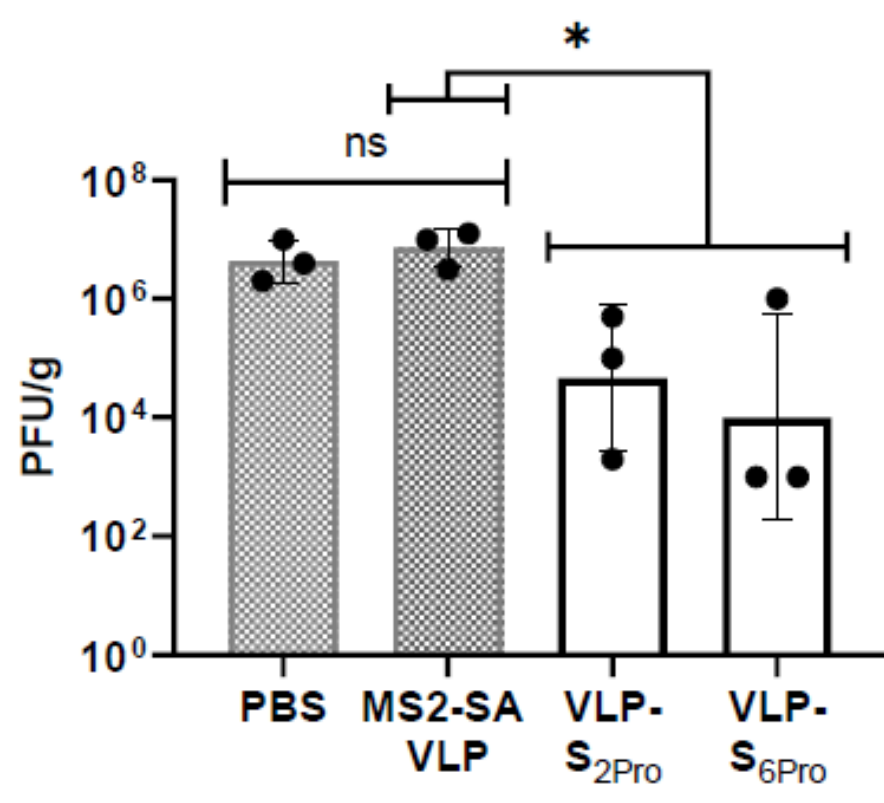

Figure 4

Protective efficacy of VLP-S. a) Schedule for vaccination of hamsters, serum collection, infection, and organ collection. b) Body weight of hamsters immunized with a single dose of either VLPS 6Pro ( circle ), VLP-S2Pro ( square ), MS2-SA VLP ( triangle), or PBS (diamond) after SARS-CoV-2 infection (mean \pm 
SD, $n=3)$. c) Viral titer in the lungs of hamsters immunized with either PBS, MS2-SA VLP, VLP-S2Pro or VLP-S6Pro three days after SARS-CoV-2 infection (geometric mean with geometric SD, $n=3$ ). $†$ - No infectious virus was detected in the lungs of hamsters immunized with VLP-S2Pro or VLP-S6Pro

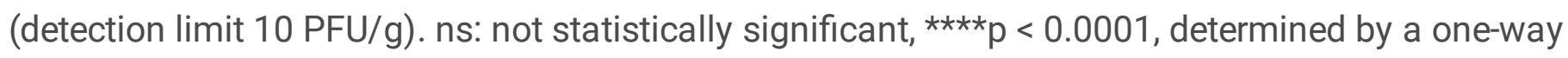
analysis of variance (ANOVA) and Dunnett post-hoc multiple comparison between groups $(a=0.1)$. Assumptions of the normality of residuals and homogeneity of variance were validated by the ShapiroWilk test and the Brown-Forsythe test, respectively. d) Viral titer in the nasal turbinates of hamsters immunized with either PBS, MS2-SA VLP, VLP-S2Pro or VLP-S6Pro three days after SARS-CoV- 2 infection (geometric mean with geometric $S D, n=3$ ). ns: not statistically significant, ${ }^{*} p<0.1$, determined by a oneway analysis of variance (ANOVA) and Dunnett post-hoc multiple comparison between groups $(a=0.1)$. Assumptions of the normality of residuals and homogeneity of variance were validated by the ShapiroWilk test and the Brown-Forsythe test, respectively. 